\title{
Multiagent based information dissemination in vehicular ad hoc networks
}

\author{
S.S. Manvi ${ }^{\mathrm{a}, *}$, M.S. Kakkasageri ${ }^{\mathrm{b}}$ and Jeremy Pitt ${ }^{\mathrm{c}}$ \\ ${ }^{a}$ Department of Electronics and Communication Engineering, Wireless Information Systems Research \\ Laboratory, Reva Institute of Technology and Management, Bangalore-560064, India \\ ${ }^{\mathrm{b}}$ Department of Electronics and Communication Engineering, Basaveshwar Engineering College, \\ Bagalkot-587102, India \\ ${ }^{\mathrm{c}}$ Department of Electrical and Electronic Engineering, Intelligent Systems and Networks Group, \\ Imperial College, London SW7 2BT, UK
}

\begin{abstract}
Vehicular Ad hoc Networks (VANETs) are a compelling application of ad hoc networks, because of the potential to access specific context information (e.g. traffic conditions, service updates, route planning) and deliver multimedia services (Voice over IP, in-car entertainment, instant messaging, etc.). This paper proposes an agent based information dissemination model for VANETs. A two-tier agent architecture is employed comprising of the following: 1) 'lightweight', network-facing, mobile agents; 2) 'heavyweight', application-facing, norm-aware agents. The limitations of VANETs lead us to consider a hybrid wireless network architecture that includes Wireless LAN/Cellular and ad hoc networking for analyzing the proposed model. The proposed model provides flexibility, adaptability and maintainability for traffic information dissemination in VANETs as well as supports robust and agile network management. The proposed model has been simulated in various network scenarios to evaluate the effectiveness of the approach.
\end{abstract}

Keywords: Multi-agents, MANETs, VANETs, information dissemination

\section{Introduction}

A Mobile Ad-hoc Network (MANET) is comprised of a group of mobile nodes which have the capability of self-organization in a decentralized fashion and without fixed infrastructure [13]. A Vehicular Ad hoc Network (VANET) is an example of a MANET where the mobile nodes are the vehicles themselves. Communication is possible between vehicles within each other's radio range as well as with fixed road side infrastructure components. The VANET concept is an integral part of the intelligent transportation system (ITS) architecture [1], which aims to improve road safety, optimize traffic flow, reduce congestion, and so on.

\subsection{Background}

VANETs are special case of MANETs. The key differences as compared to MANET environment are following.

\footnotetext{
${ }^{*}$ Correspending author. E-mail: sunil.manvi@ revainstitution.org.
} 
- Components building the network are vehicles.

- Restricted mobility constraints.

- Extremely high mobility and time-varying vehicle traffic density.

- Most of the vehicles provide sufficient computational and power resources, thus eliminating the need for introducing complicated energy aware algorithms.

- Vehicles will not be affected by the addition of extra weight for antennas and additional hardware.

Most of the protocols designed for MANETs may not be directly applied to VANETs, since they may not take into account high mobility and restricted mobility constraints (roadways). Some parameters which have to be mainly concentrated in VANETs for protocol design are extremely high mobility, restricted movements, fast topology changes and time varying vehicle traffic density.

\subsubsection{Issues in VANETs}

VANET raises several interesting issues with regard to Media access control (MAC), Mobility management, Data aggregation, Data validation, Data dissemination, Routing, Network congestion, Performance analysis, Privacy and Security [29,40].

- Media access control (MAC): VANET MAC protocols may not be required to significantly consider power constraints or time synchronization problems. However they have to consider the fast topology changes, as well as the different kinds of applications for which the transmission will be established. Moreover, VANET MAC protocols must reduce the the medium access delay and increase the reliability, which is important in case of safety applications.

- Mobility management: Since vehicles are highly mobile and change their point of network attachment frequently while accessing Internet services through gateways, it is advisable to have some mobility management schemes that take care of vehicle mobility and provide seamless communication. Mobility management has to meet the following requirements: seamless mobility (communication must be possible irrespective of vehicle position), low hand-off latency, support IP V6 and scalable overheads.

- Data aggregation: The vehicles have to pass on the data sent by the neighbors to other neighbors in its coverage area. This increases the number of packets to be sent by a vehicle. Therefore, data aggregation techniques must be applied to reduce such overheads. Data aggregation is an interesting approach which reduces the number of packets transmitted drastically by combining several messages related to the same event into one aggregate message. For example, the records about two vehicles can be replaced by a single record with little error, if the vehicles are very close to each other and move with relatively the same speed.

- Data validation: A vehicle may send the observed data directly (assuming that a vehicle always trusts the data it has gathered itself) to its neighbors. Sometimes malicious vehicles may send the incorrect information to confuse the users. For example, a malicious node may send the false accident information and divert all the vehicles on other roads, which may lead to traffic congestion. In such situation, data validation techniques must be applied before passing on the received information to other nodes.

- Data dissemination: Data dissemination can be defined as broadcasting information about itself and the other vehicles. Each time a vehicle receives information broadcasted by another vehicle, it updates its stored information accordingly, and defers forwarding the information to the next broadcast period. The dissemination mechanism should be scalable, since the number of broadcast messages is limited, and they do not flood the network. VANET characteristics like high-speed node movement, frequent topology changes, and short connection lifetime especially with multihop paths need some typical data dissemination models for VANETs. 
- Routing: Since the topology of the network is constantly changing, the issue of routing packets between any pair of nodes becomes a challenging task. Most protocols should be based on reactive routing instead of proactive. Multicast routing is another challenge because the multicast tree is no longer static due to the random movement of nodes within the network.

- Network congestion: Congestion control in VANETs is a challenging issue. The Internet is based on an end-to-end paradigm, where the transport protocol (e.g. TCP) instances at the endpoints detect overload conditions at intermediate nodes. In case of congestion, the source reduces its data rate. However, in VANETs the topology changes within seconds and a congested node used for forwarding a few seconds ago might not be used at all at the point in time when the source reacts to the congestion.

- Performance analysis: The cost of deploying and implementing designed schemes for VANETs in the real world is high. Hence there is a need for development of effective simulators to evaluate the performance of protocols for VANETs before deployment. A VANET simulation platform must support vehicle to vehicle as well as vehicle to base station communications in various road conditions, traffic conditions and mobility patterns. Simulation may also use collection of mobility traces and network statistics to experiment on a real vehicular network.

- Privacy and security: VANETs demand a thorough investigation of privacy related issues. On one hand, users of such networks have to be prevented from misuse of their private data by authorities, from location profiling and from other attacks on their privacy. On the other hand, system operators and car manufacturers have to be able to identify malfunctioning units for sake of system availability and security. Wireless link characteristics introduce reliability problems because of the limited wireless transmission range, broadcast nature of the wireless medium (e.g. hidden terminal problem), mobility-induced packet losses, and data transmission errors.

\subsubsection{Applications of VANETs}

With the increase of portable devices as well as progress in wireless communication, VANET is gaining importance with the increasing number of widespread applications. Some of the important applications of VANETs are as follows.

- Message and file delivery: This application focuses on enabling the delivery of messages and files in a vehicular network to the target receivers with acceptable performance. A group of applications, such as accident and road construction warning systems, require the network protocols to forward messages from a sender to only relevant receivers based on the location and driving direction. Also, safety applications are time sensitive and should be given priority over non-safety applications.

The critical issue with respect to message and file delivery is the enforcement of cooperative communications among VANET nodes. Uncooperative behavior in VANET, such as message modification and dropping, could substantially lower the message delivery ratio and further affect the safety of other vehicles. Therefore, reliable mechanism are needed to promote cooperation in VANET for reliable message and file delivery.

- Location-dependent services: Vehicles equipped with GPS providing accurate geographical position must be integrated into services, such as the location of the nearest facilities like fuel stations, parking zones, entertainment places and restaurants, etc. One can imagine this being further integrated into either telemetry (e.g. distance to empty) or higher-level recommender systems providing feedback on facilities from other VANET nodes (e.g. quality, convenience, etc.).

In VANETs, knowledge of the real-time position of nodes is an assumption made by most protocols, algorithms, and applications because of GPS receivers. But as VANETs advance into critical areas 
and become more dependent on localization systems, GPS shows some undesired problems such as not always being available or not being robust enough for some applications. Hence there is a need for development of new localization techniques to overcome GPS limitations.

- Internet connectivity: Typically established Internet applications like web browsing, e-mail, chat, mobile commerce, and entertainment can be provided by using road side infrastructure along with VANETs that are connected to fixed infrastructure like Internet and other required networks.

Vehicles can gain short connections to the Internet by using roadside gateways. A significant part of the connection time is the time required for acquiring an IP address via Dynamic Host Configuration Protocol (DHCP). Due to vehicle's speed (mobility) and the gateway coverage area, new mechanisms are needed to reduce the overhead of obtaining an IP address. Hence devising a protocol for Internet connectivity application is required, which takes care of improvement in efficiency, reduction in latency, and increase in vehicle connectivity.

- Information and Warning Functions (IWFs): The purpose of IWFs is to warn vehicles to approach a possibly dangerous area with caution like dissemination of road information (including incidents, congestion, surface condition, etc.) to vehicles distant from the subjected site.

VANETs present a unique challenge in enabling IWFs because of extremely high mobility and rapidly changing topology. However, this mobility is constrained in motion due to the existence of roadways and can therefore be cleverly exploited for propagation of IWFs.

- Co-operative assistance systems: Co-operative assistance systems are a key issue in the visionary field of accident-free driving. For example, coordinating vehicles at critical points such as blind crossings (a crossing without light control) and highway entries.

In co-operative assistance systems, vehicles should be enabled to foresee critical driving conditions and therefore inform their drivers in timely manner. In this context, direct communication among vehicles that form a spontaneous ad hoc network will complement on-board sensor systems, enabling a new paradigm in driving assistance: collaborative and predictive situation-awareness.

- Safety services: Safety applications include emergency breaking, accidents, passing assistance, security distance warning, and coordination of cars entering a lane. Furthermore, sensors embedded in the car engine and elsewhere could be used for exchanging information, either with the onboard computer of the vehicle itself or with higher-end vehicles with sophisticated computing and communication abilities, for diagnostic purpose. This could facilitate preventive maintenance and minimizes road breakdowns.

Communication between vehicles and road side infrastructure plays an important role for the safety services. Traffic lights or road signs could be equipped with a communication device to actively inform vehicles in the vicinity. Hence, drivers can receive information on traffic flow, road conditions or construction sites directly from the respective road side infrastructure. In addition, static hazard areas, e.g. construction sites, could be equipped with a road side infrastructure to warn surrounding vehicles.

- Traffic monitoring and management services: In such type of services all vehicles are part of a ubiquitous sensor system. Each vehicle monitors the locally observed traffic situation, such as density and average speed, using onboard sensors and the results are transferred via wireless data-link through the network.

For traffic monitoring and management services, an important problem that has to be addressed is exchange of traffic information among vehicles in a scalable fashion. In some applications, information is disseminated proactively using broadcast (push model), while in others the information is obtained on-demand (pull model). Thus selecting an appropriate model is important for such kind of services. 
- Other advanced services: It is possible to imagine other interactions between VANET nodes and the road infrastructure, for example traffic calming measures (including automatic speed limiters), intelligent road signs, and tolling (congestion charges for entering urban areas at peak times, cargo monitoring, and so on.)

For such kind of other advanced services, broadcast messages will play a larger role than the use of unicast messages in a VANET. But the problem is that no retransmission is possible for failed broadcast transmissions since they cannot be detected.

\subsection{Related works}

Several works are reported in the literature that deal with ad hoc networks and their applicability in VANETs. This section presents some of the important works. The work given in [18] describes a system called Ad Hoc City, which is a multi tier wireless ad hoc network routing architecture for general-purpose wide-area communication. The backbone network in this architecture is a mobile multihop network, composed of wireless devices mounted on mobile fleets such as city buses or delivery vehicles.

The work given in [25] addresses the issues pertaining to medium access control schemes in highly dynamic automotive networks that reduce latency and perform reliable communication. It also describes a distributed positioning algorithm, called the kernel algorithm, suited for asynchronous ad hoc wireless networks under complexity constraints. Traffic congestion avoidance by disseminating traffic information through peer to peer networks based on WiFi technology is studied in [34].

A mixed mode wireless LAN comprising of infrastructure and ad hoc mode operations is presented in [15] where MANETs are connected through several base stations. The work given in [19] presents an autonomous, self-organizing and decentralized configuration and management system for a group of base stations in wireless networks. The individual base stations aggregate and share network information. A distributed algorithm computes a local configuration at each base station based on the shared information.

For a given communication graph, the method of introducing relay nodes is discussed in [30]. Transmitting ranges are assigned to the nodes such that the cost of range assignment function is minimal over all connecting range assignments in the graph. In this work, the algorithm does not assume that communication graph to be a unit disk graph. The output of the algorithm is the minimal Steiner tree on the graph that consists of terminal (original) nodes and relay (additional) nodes. The output of approximation is the range assignments to the nodes.

A dynamic clustering solution which is distributed in nature, handles the cluster management by taking into account practicalities like packet losses etc., and integrates with a routing module [28]. A security concept based on a distributed certification facility is described in [21], where a network is divided into clusters with one cluster head node for each cluster. These cluster head nodes execute administrative functions and hold shares of a network key used for certification. The work given in [8] introduces a scalable service discovery protocol for MANETs, which is based on the homogeneous and dynamic deployment of cooperating directories within the network. A congestion control method with dynamic clustering for variable topology and link qualities is discussed in [20].

A dynamic probabilistic approach that dynamically fine-tunes the rebroadcasting probability according to the number of neighbor nodes distributed in the ad hoc network for routing request packets (RREQs) is presented in [3]. Stealth attacks in the context of three common types of wireless networks, namely ad hoc networks, hybrid networks, and sensor networks are discussed in [22]. AODV-SEC, a new secure routing protocol has been presented and thoroughly evaluated in [31]. This protocol takes into account the special security and performance requirements of MANETS, based on a single PKI. A realistic city 
mobility model was used to examine the performance of routing protocols AODV, DSR, FSR and TORA by considering urban traffic scenarios in VANETs [35].

Sensor nodes are used to provide variety of services in VANETs [24]. A protocol architecture for VANETs describing layer-wise functioning of protocols used for vehicle communication is given in [10]. The work given in [11] presents a multi-agent system for monitoring and assessing air quality attributes, which uses data coming from a meteorological station. A community of software agents is assigned to monitor and validate measurements coming from several sensors, to assess air-quality, and, finally, to fire alarms to appropriate recipients, when needed. Mobile agent based organization is discussed in [9]. The idea is to model the Internet as a multiplicity of local and active organizational contexts, intended as the places where coordination activities of application agents occur and are ruled. The work given in [42] presents agent based platform for VANETs.

Recent developments in collision avoidance/warning systems and automation of vehicle lateral control tasks are discussed in [4]. This work explains the initiatives for automation in different levels of transportation system with a specific emphasis on the vehicle-level automation. Auto- and cross-correlation properties of various optical spreading codes are compared and the infrared direct sequence spread spectrum (DSSS) inter-vehicle ranging and vehicle-to -roadside communication systems are proposed in [16]. Simulations and mathematical models for inter-vehicle communication (IVC) architectures, including some security implications are discussed in [17]. Directional Propagation Protocol (DPP), which uses the directionality of data and vehicles for information propagation is discussed in [45]. DPP is comprised of three components; a Custody Transfer Protocol (CTP), an Inter-Cluster Routing Protocol, and an Intra-Cluster Routing Protocol. A survey of existing secured routing protocols, that are suitable for VANETs are discussed in [7].

An efficient lightweight support for proactive urban monitoring based on the primary idea of exploiting vehicle mobility to opportunistically diffuse summaries about sensed data is discussed in [46]. A dynamic collision avoidance component for the standard vehicle-following approach has been introduced in [37]. The elastic-band framework is used to modify the initial path of the leader vehicle.

\subsection{Our contributions}

The proposed model is motivated by observing some inherent drawbacks of existing information dissemination mechanisms of VANETs like: high latency, high bandwidth consumption, less robust to dynamic topology, and low scalability. This paper proposes multiagent based information dissemination in VANETs, which uses a set of static and mobile agents in VANET agency. VANET agency is further classified into three categories: Vehicle agency, Base station agency, and Regional transport station (RTS) agency.

Three types of agents are used in the vehicle agency: Vehicle Manager Agent (static), Alarm Agent (mobile), and Service Discovery Agent (mobile). Vehicle Manager Agent is deployed at each vehicle, which creates traffic knowledge base, all the set of agents within the vehicle agency, and synchronizes the interactions of all the agents within the vehicle. Traffic knowledge base works on the principle of blackboard architecture, which is used for communication among agents. Alarm agent travels around the network to disseminate the critical information during the critical situations. Service discovery agent travels in the network to search for the required services as desired by the vehicle user.

Three types of agents are used in the base station agency: Base Station Manager Agent (static), Service Agent (static), and Advertisement Agent (mobile). Base station manager agent is a norm-governed agent, which maintains cluster knowledge base and synchronizes all the agents that are associated with base 
station. Cluster knowledge base comprises of critical events within cluster. Service agent is responsible for collecting the services information from the service providers. Advertisement agent is a mobile agent which roams in the network and collects the information.

Two types of agents are used in the RTS agency: RTS Manager Agent (static), Service Discovery Agent (mobile). RTS manager agent maintains knowledge base and synchronizes all the agents that are associated with regional transport station. Knowledge base comprises of traffic related information. Service discovery agent travels in the network to search for the required information as desired by the regional transport station.

Our contributions in the paper are as follows:

- We introduce the concept of usage of software agents for information dissemination in VANETs. The focus of this paper is to exploit the features of software agents to overcome the limitations of VANET.

- We proposed two-tier architecture for the information dissemination model of VANETs. With this architecture VANET can be operated in any of the following modes: WLAN/cellular, ad hoc, and hybrid mode (combining of WLAN/cellular and ad hoc). This architecture is flexible, and scalable.

- We show that the proposed model is efficient for three kind of applications: Information search and access, Critical information dissemination, and location dependent services.

- We evaluate the roles of software agents that significantly impact VANET performance, including the less information saturation time and latency, and low bandwidth utilization for dense VANET.

The rest of the paper is organized as follows. Section 2 presents the role of software agents in VANETs. Section 3 discusses the proposed agent based information dissemination model in VANETs. Simulation model and results are presented in Section 4. Section 5 presents the benefits of using agent technology. Finally we conclude in Section 6.

\section{Software agents}

The traditional programming paradigm uses functions, procedures, structures and objects to develop a software for performing a given task. This paradigm does not support development of flexible, intelligent and adaptable softwares, and also does not facilitate all the requirements of Component Based Software Engineering (CBSE) [5,6,23,26,38,44]. In recent developments, agent technology is making its way as a new paradigm in the areas of artificial intelligence and computing, which facilitates sophisticated software development with features like flexibility, scalability and CBSE requirements $[32,43]$.

Agents are the autonomous programs which sense the environment and acts upon the environment using its knowledge to achieve their goals $[33,27,12,36]$. The agent environment generally referred as a host system, network, a user via a graphical user interface, a collection of other agents, or perhaps all of these combined. Agents are classified as single agent and multiagent systems. Single agent systems comprise of a single agent interacting with resources, humans and other processes to perform a dedicated task. MAS comprises of set of agents that interact, cooperate, and coordinate with each other to perform a set of tasks or a dedicated tasks. Mobile agents are multiagent systems, which roam in the network to achieve their goals [39].

Now, we will briefly describe comparison of mobile agent based approach with simple message passing, which could have been used for information dissemination. Message passing is a form of communication used in concurrent computing, parallel computing, object-oriented programming, and interprocess communication. Mobile agents allows processes to migrate from computer to computer, for 
processes to split into multiple instances that execute on different machines, and to return to their point of origin. Mobile agent computing, considered as a special case of message passing, attempts to move computations as close as possible to the data and makes efficient use of the bandwidth by considerably decreasing the number of messages exchanged between cooperating applications.

The advantages offered by using Mobile Agent based Network Management (MANM) include network traffic reduction, intelligence, automation, fault-tolerance, and robustness. In the proposed system, data dissemination can also be performed with message passing (i.e., without mobile agents). However the agent based coordination mechanism used here is particularly attractive for collaborations in intermittently connected environments. While a user is disconnected from the environment, agents can migrate in the connected backbone network to perform coordination actions at other nodes.

Another advantage of using mobile agent arises while waiting to ensure the precondition for a coordination operation at a remote node. In contrast to simple message passing approach, the mobile agent based approach obviates the need of either maintaining a connection or making repeated attempts until the required precondition is satisfied.

In summary, the advantages of using mobile agents as compared with the simple message passing approach are as follows.

- Mobile agents reduces the network load. Simple message passing approach often uses more bandwidth.

- Mobile agents can operate directly on mobile nodes thus avoiding network latency while operating locally. This is most relevant in VANETs where network latency is a prime issue.

- Mobile agents interact with their environment and adapt themselves.

- Mobile agents can be embedded with intelligence in order to perform data aggregation and validation.

- Mobile agents can execute in asynchronous and autonomous fashion. This autonomy along with platform and system independence make them ideal for building reliable and robust applications, and can thus deal with the environment reacting dynamically to changes like VANETs.

- The mobile agent code can encapsulate the protocol. When a protocol is upgraded, only the mobile agent has to be altered.

- Mobile agents react dynamically and autonomously to the changes in their environment, which makes them fault tolerant.

\subsection{VANET features that forces to use software agents}

Network management, service provisioning and information distribution to (multimedia) applications in VANETs is intrinsically difficult. Hence, it is required to solve these problems. The proposed solution is a two-tier architecture which integrates mobile agents and norm-aware agents, the idea being to provide both a rapid response to changes in a high speed environment with rational deliberation about social behavior, contracts and sub-ideality [2,14,41].

As stated above, a VANET is a temporary association of mobile nodes which manage themselves independently of any fixed support infrastructure (there may be connections to fixed infrastructure nodes but these do not affect network management decisions). They are self-created and self organized, are inter-connected by mutli-hop wireless paths and operate in strictly peer-to-peer fashion. The benefits include bandwidth re-use, low-cost (or even zero-cost) rapid deployment, and intrinsic fault-tolerance. The drawbacks are, as indicated, that problems of network management, service provisioning and information distribution are exacerbated, because the dynamic network topology renders centralized solutions based on complete information impossible. 
MANETs, as originally conceived, were intended to be transient networks, supporting short-lived, opportunistic and spontaneous networks rather than long term inter-operation. VANETs, on the other hand, are expected to have a much longer life-span, and have a unique property in that, in one sense the network is always the same every time a vehicle joins it (e.g. on a commuting route), but each of the nodes which constitute the network are all different. The issue then is that if long-term operation is required, the usual networking solution is to create a backbone (for efficient packet routing); the issue then is how to select the vertebrae when the network nodes are constantly changing (even if the network stays the same). The field of mechanism design in multiagent systems (the process of designing a choice mechanism that translates expressed preferences into a decision) suggest several solutions (e.g. auctions, voting, etc.), but in any solution agents require accurate, timely information in order to make bids, cast votes, and so on.

To address these issues, there are two requirements. The first requirement is for snap decisions taken at the local point of need taken from an individual perspective. To support this, we suggest using mobile agents. The basic idea behind mobile agents is to move code across a network from one node to another. This is technically feasible if basic requirements for secure communication and code execution can be met, and a common execution language which supports process persistence. Note these processes are agents if they preserve autonomy of state and decision-making, unlimited outbound communications, and asynchrony of computation. The general motivation for using mobile code includes: the code footprint is small compared to data; the process is operating in a dynamic decentralized environments; for efficiency, code can be transported on packets that will be sent anyway (autonomic communications), and the environment demands both location transparency (logical distribution of responsibility and control) and location contingency (who is actually doing the work is important). These are all features of the VANET environment.

The second requirement is for considered deliberation from a global perspective, including each other, based on informal social relations (e.g. quid pro quo) or formal commercial relations (e.g. contract). Therefore cooperation in VANETs needs notions of trust, selfishness, fairness, judgment, punishment and forgiveness, plus a balance between local plus global decision-making which needs notions of delegation, mandate, and authorization, and so on. All of the above are socio-legal concepts and are amenable to normative specification, and these specifications can be used by norm-aware agents. Any set of interacting agents whose behavior is regulated by norms can be considered a norm governed system, where a norm is a rule which prescribes organizational concepts like (institutional) power permission, obligation, sanction, and other more complex relations. This approach to multi-agent systems has its roots in the study of legal, social and organizational systems, while formal analysis often use some version of deontic logic which provides route to automation. Such automation offer formal definitions of responsibility and control, interoperability (as defined in terms of external, executable specifications), and reasoning about sub-ideal states of the system (i.e. detecting and recovering from faults in the system, which are to be expected in probabilistic systems like VANETs.)

\section{Agent based information dissemination model}

Considering all the properties of the agent systems, we propose to implement the two-tier agent architecture over a three-tier network architecture as shown in Fig. 1.

The first tier contains the vehicles which are communicating themselves on the road that are within a given cluster (a VANET). A cluster is formed based on radio range coverage of the road-side base station. In the second tier, a set of clusters are there in which each cluster comprises of base stations (C1 


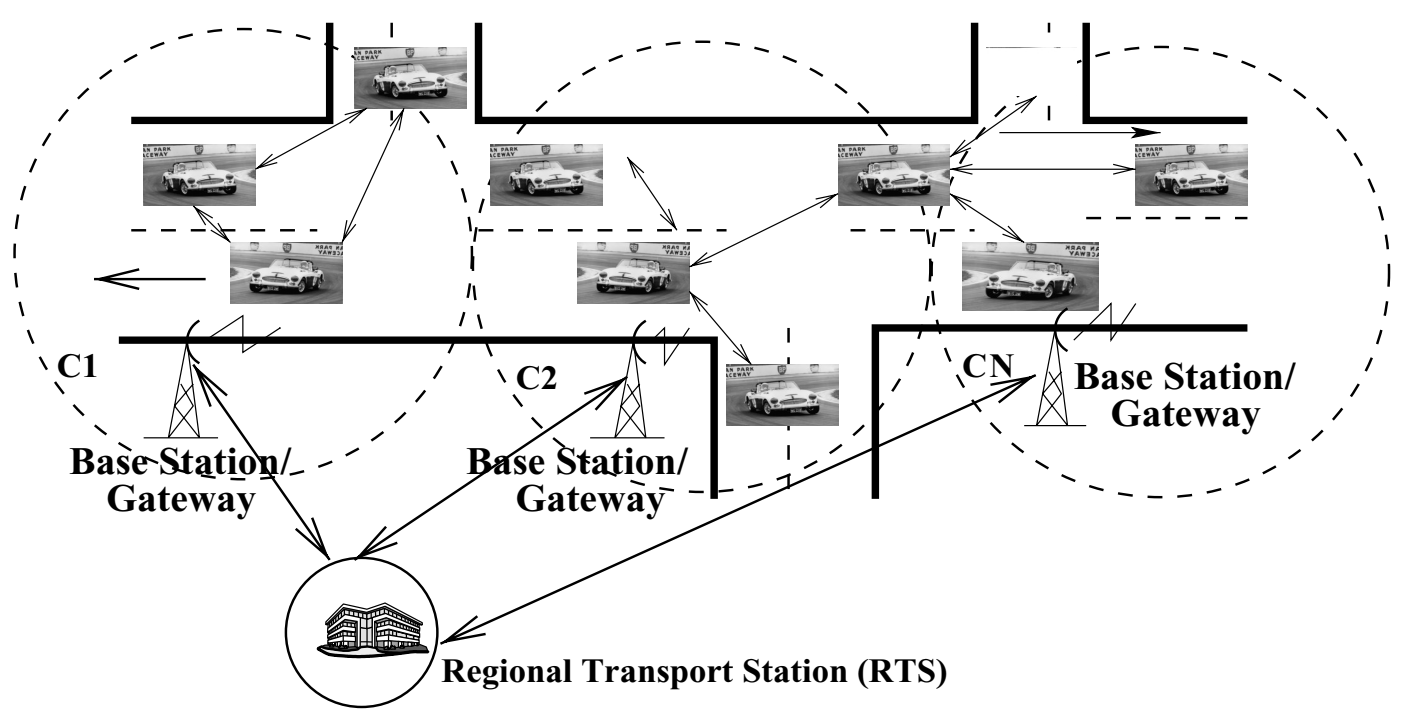

Fig. 1. Three tier VANET.

to $\mathrm{CN}$ clusters). The clusters may communicate with each other by using base stations. The third tier is the regional transport station (RTS), owned by a private body or government agency to monitor the entire transportation infrastructure and offer the relevant services to the vehicles on the road.

\subsection{VANET agencies}

The following agencies are employed to perform communication among vehicles, base station, and regional transport station: vehicle agency, base station agency and regional transport station agency.

\subsubsection{Vehicle agency}

Vehicle agency works on the principle of black board architecture (for inter agent communication and coordination). The components of a vehicle agency are depicted in Fig. 2, showing the interactions by using numerical value on the arcs. Vehicle agency consists of a set of agents such as Vehicle Manager Agent (VMA), Alarm Agent (AA), and Service Discovery Agent (SDA). These agents are responsible for collecting and disseminating traffic information. The vehicle agency also consists of a traffic knowledge base (TKB) that works on the principle of blackboard architecture, which is used for communication among agents.

- Vehicle Manager Agent (VMA): It is deployed at each vehicle. This is a static agent which creates traffic knowledge base (1 in Fig. 2), all the set of agents within the vehicle agency ( 2 and 3 in Fig. 2), and synchronizes the interactions of all the agents within the vehicle. This agent directly communicates with the cluster base station to get/disseminate the relevant information/services especially in critical situations. All other agents are triggered by this agent to perform a specific dedicated task.

VMA is a norm-aware agent that incorporates trust, selfishness, fairness, judgment and punishment. VMA tries to be selfish by gathering almost all the information about critical events and other services by using certain selfish norms such as giving more importance to multimedia services or recent road maps, etc. If VMA receives more number of critical events in the network, it fairly sorts 


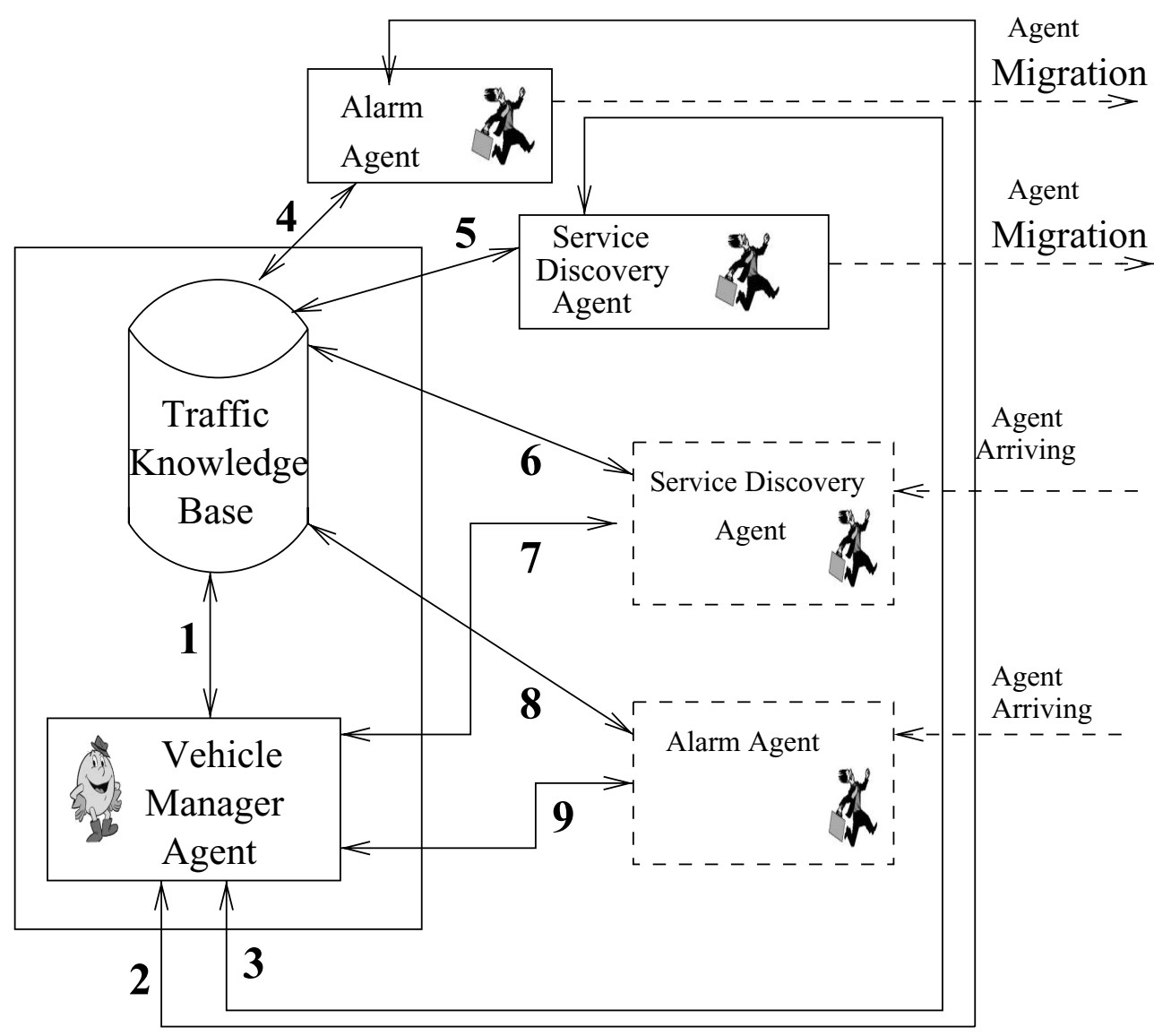

Fig. 2. Vehicle agency.

out all the events and distributes to neighbors and road-side gateway. If an event is received more than once or after an expired time of the event, VMA makes judgment whether to consider that event or not. At any given time if VMA finds any agent from a malicious node it punishes that agent by not accepting or communicating with that agent.

This norm-governed heavy weight agent also addresses the following.

* Decides the critical situations based on certain rules and code of conduct. The situations may be monitored through either sensors or neighboring AAs or the vehicle user. Rules may be based on degree of worsened road conditions, nature of accident, level of fuel, tire pressure, reliability of neighboring nodes, etc.

* Provides access to internal services such as audio files, road information, data, etc., to other vehicles based on certain permissions.

- Alarm agent: It is a mobile agent created by VMA ( 2 in Fig. 2), that travels around the network by creating its clones (a clone is a similar copy of the agent with different destination addresses) to disseminate the critical information during the critical situations. Examples of critical situations are accident, traffic jam, bad weather conditions, fuel status, road maintenance, etc. It also informs the VMA and updates the TKB (4 in Fig. 2) when cloning to other vehicles. 


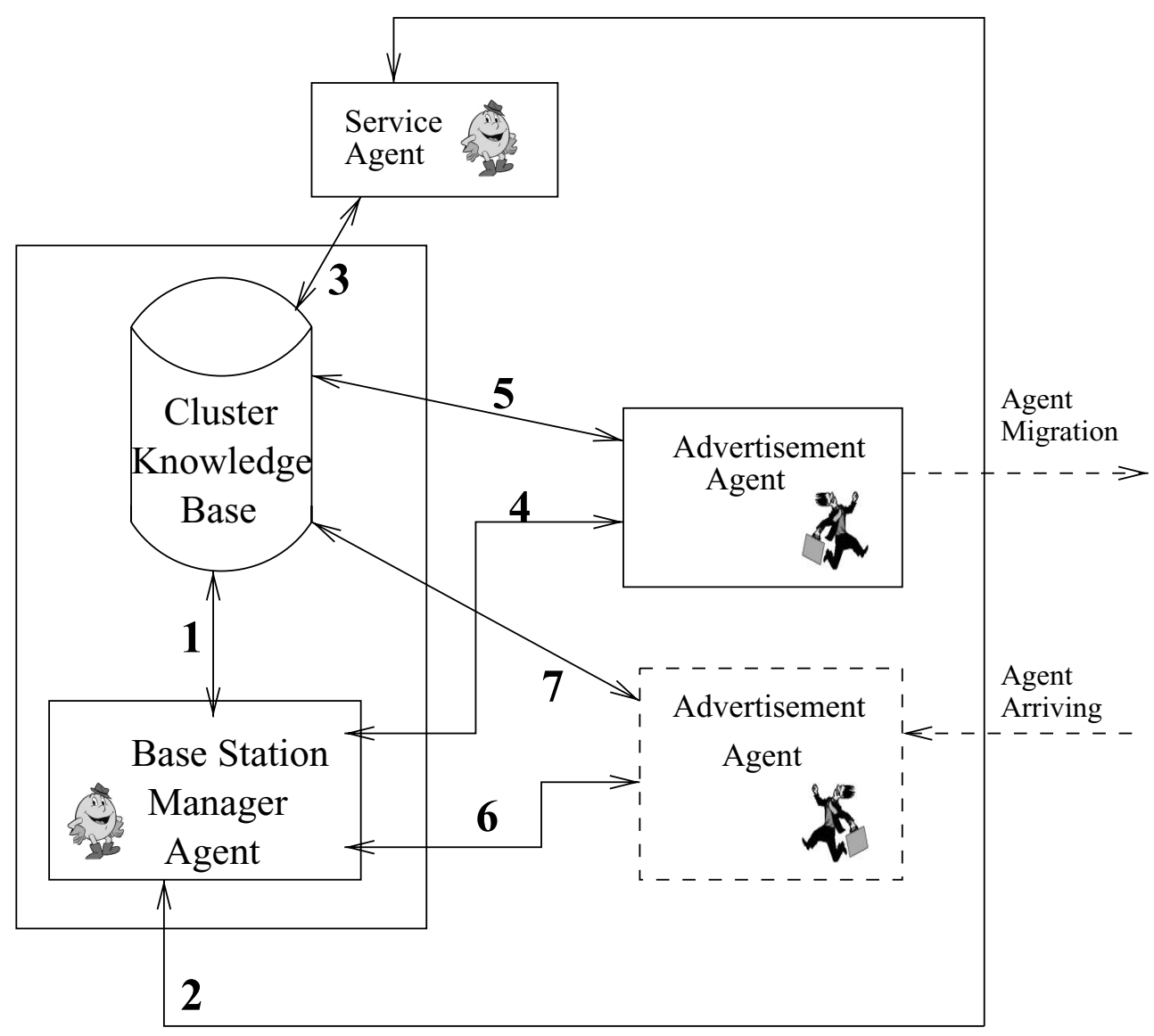

Fig. 3. Base station agency.

This agent may also arrive to a vehicle from other vehicles to exchange the critical event information. In the process, it interacts with VMA and updates TKB as well as read the unknown critical events from the TKB to carry further to other vehicles (8 and 9 in Fig. 2)

- Service discovery agent: It is a mobile agent created by VMA (3 in Fig. 2) which travels in the network to search for the required services as desired by the vehicle user. The services may be road maps, traffic density maps, Internet services, and location aware services (commerce, entertainment, parking, fuel stations, etc.). The agent also updates the TKB with services discovered (5 in Fig. 2). The arriving SDA interacts with VMA and updates the TKB with services it has discovered in the visited vehicle agency as well as reads the unknown services from TKB to carry further in it's travel (6 and 7 in Fig. 2).

- Traffic knowledge base: It comprises of information of critical events such as accidents, traffic density and the services available in the vehicle, services accessed, recently accessed road maps, etc.

\subsubsection{Base station agency}

The agency in the base station comprises of following components: Base Station Manager Agent (BSMA), Service Agent (SA), Advertisement Agent (ADA) and Cluster Knowledge Base (CKB). The components and their interactions are depicted in Fig. 3. 
- Base station manager agent: It is a static norm-governed agent deployed at each base station, which maintains cluster knowledge base and synchronizes all the agents that are associated with base station (1, 2 and 4 in Fig. 3). It incorporates trust, selfishness, fairness, judgment and punishment. BSMA trusts other agent that have authenticated certification. Selfishness is incorporated in BSMA, i.e., BSMA tries to gather much of its cluster information and the neighbors information as per the service interests. The critical events happened in area are judged by the BSMA for their truthfulness based on information gathered by different agents pertaining to a same area. BSMA fairly sorts out all the events and distributes to all other clusters by using passing vehicles.

It regularly updates the CKB with the visited vehicles and its services information by interacting with VMA of each vehicle. Also computes the traffic density maps, adverse road conditions and updates the CKB. Critical information received from VMAs in its cluster is sent to other BSMAs. This agent is responsible for communicating information with VMAs, BSMAs and RTSMA.

- Service agent: It is a static agent responsible for collecting the services information from the service providers of the cluster and regularly updates the CKB (3 in Fig. 3). SA also reads from CKB before updating to avoid duplicate information. It also broadcasts any critical information available with it to the vehicles within its cluster upon notification from other BSMAs.

- Advertisement agent: It is a mobile agent which roams in the network and informs the visited VMAs about the auctions, special exchange offers, ticket reservations, etc. It may interact with the user and get the information about his participation in auctions or booking tickets or any such tasks as the user wishes. It regularly updates the CKB by interacting with BSMA (5 in Fig. 3).

An arriving AA from other vehicles interact with BSMA and performs reading and updating of CKB (6 and 7 in Fig. 3).

- Cluster knowledge base: It comprises of information such as critical events within cluster, services available in cluster, visited vehicle information, traffic density maps, road conditions, location aware services, advertisements, etc.

\subsubsection{Regional transport station agency}

The agency in the regional transport station comprises of following components: RTS Manager Agent (RTSMA), Service Discovery Agent (SDA), Knowledge Base (KB). The components and their interactions are depicted in Fig. 4.

- Regional transport station manager agent: It is a static norm-governed agent deployed at regional transport station, which maintains knowledge base and synchronizes all the agents (1 and 2 in Fig. 4) that are associated with regional transport station. It computes the traffic density maps, adverse road conditions and updates the KB.

- Service discovery agent: It is a mobile agent which travels in the network to search for the required information as desired by the regional transport station. The agent also updates the KB with collected information (3 in Fig. 4). An arriving SDA will interact with RTSMA and performs reading and updating of KB (4 and 5 in Fig. 4).

- Knowledge base: It comprises of information such as traffic, critical events, road conditions, list of services available in its entire area, etc.

Practical implementation of the proposed scheme needs the following: 1) the vehicle must be equipped with a computational device comprising a real time operating system, wireless transceiver unit with dynamic ranges, GPS unit, speed sensing unit, inter-vehicle distance monitoring unit, cameras (optional), fuel sensing unit, human interface, embedded tire air sensing unit, database manager, an agent platform with set of static and mobile agents; 2) the base station must have a computational unit, wireless transceiver 


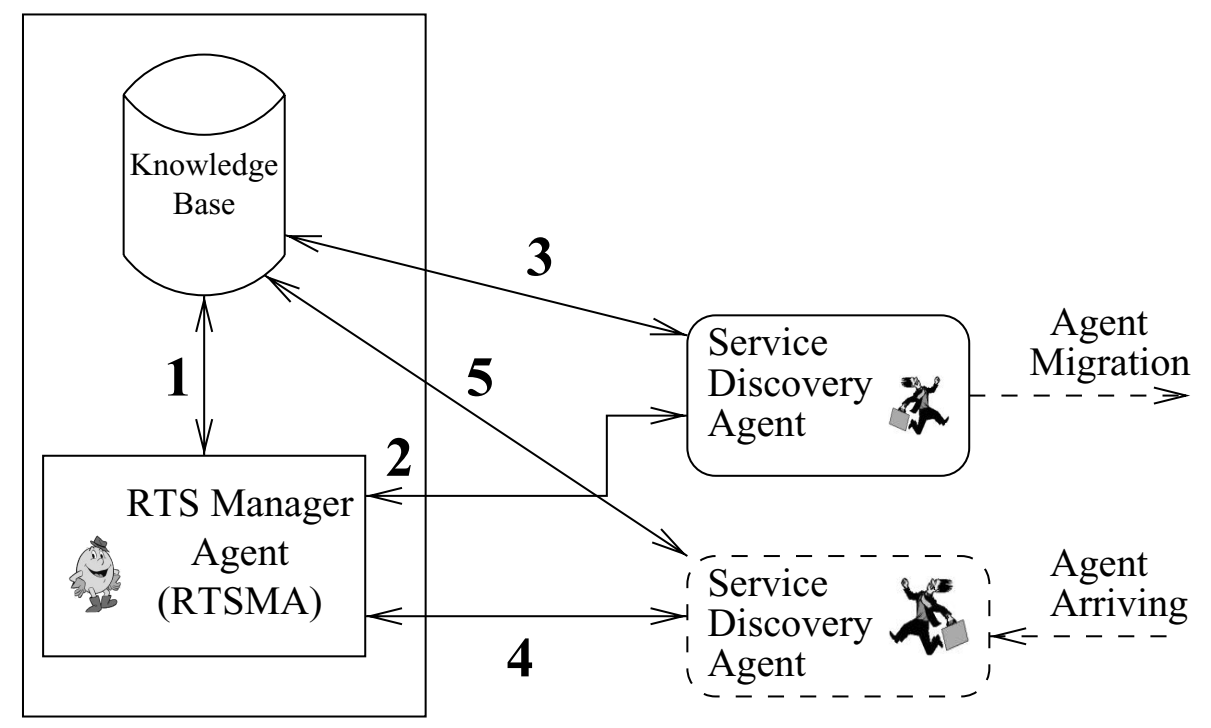

Fig. 4. Regional transport station agency.

unit, real time operating system, agent platform, cameras and database manager; 3) environment and road condition sensors are connected to base station; and 4) Transport agency comprises of computational unit, wireless transceiver unit with dynamic ranges, real time operating system, database manager, agent platform, human interface and Internet connection.

\subsection{Vehicle communications}

From the proposed network architecture, we can categorize the information dissemination and network management model into four parts: vehicle to vehicle communication, vehicle to base station communication, cluster to cluster communication and cluster to regional transport station communication.

\subsubsection{Vehicle to vehicle communication}

Vehicle to vehicle communication resembles peer to peer network architecture. The vehicle (VMA) needing information creates the SDA to its neighboring vehicles. SDA migrates to neighboring vehicle and communicates to VMA through TKB. If the required information is available in TKB of the neighboring vehicles SDA sends the information to the VMA. If the information is not available with the neighboring vehicle, SDA clones from its place and moves to second degree neighbors and so on within the cluster.

\subsubsection{Vehicle to base station communication}

A fixed infrastructure comprised of (at least) a number of base stations strategically positioned in close proximity to the highways is necessary to facilitate the upload/download of data from/to the vehicles. Each base station covers a cluster. We assume that several sensors information are input to the base station. Information could be traffic density, vehicle types, adverse road conditions, etc. If information is not available within the cluster, VMA generates SDA to its base station. In case SDA gets information at neighboring BSMA, it returns to its created VMA. 


\subsubsection{Cluster to cluster communication}

Clustering provides a method to disseminate the traffic information as well as provide varieties of services. Whole network is divided into self-managed groups of vehicles called clusters. These clusters continually adapt themselves to the changing network topology and new cluster configurations. Communication between the clusters will take place with the help of BSMAs located in base stations that are fixed on the road side. Here SDA clones itself to neighboring clusters by communicating with its cluster BSMA under certain norms.

Base station acts as a cluster head node for communication among vehicles. Cluster is adapted to the network dynamics with the help of BSMA located in a base station. BSMA is responsible for updating the network variations such as the nodes entering/leaving its area, service available in nodes, advertisements, etc.

\subsubsection{Cluster to regional transport station communication}

RTS consists of complete information of the transportation infrastructure, which is accumulated from various cluster BSMAs. BSMAs of the clusters communicate with the RTS manager. RTS manager periodically constructs a overall picture of the road ways in terms of traffic, critical events, road conditions, etc., and constructs a road map and distributes to the BSMAs. It also prepares list of services available in its entire area and stores in its knowledge base, which may be used by SDA to discover the services. When RTS wants to communicate with any cluster in the network, RTS manager sends the information to BSMA. BSMA updates its CKB and informs SA to advertise the message through ADAs in the entire network.

\subsection{Application scenarios}

In this section we illustrate the operation of the system to realize some of the application scenarios. We assume that an agent platform exists in vehicles, base stations and regional transport station. However agents communicate with each other by using traditional exchange mechanisms if an agent platform is not available in any of the components of VANET. The agent platform provides following services: agent creation, mobility, communication, security and fault tolerance.

\subsubsection{Information search and access}

Access to fixed infrastructure is essentially using the VANET to connect to any computer terminal in the car to the Internet; however, we seek to optimize performance by caching regularly-accessed information in the cluster. Required information is first searched within the cluster, i.e., by polling the BSMA and the VMAs. If the information is not available within the cluster, it searches in the neighboring cluster. The expected information cached would be road maps, traffic density maps, articles, etc.; of course other Internet services e.g. VoIP would also be available.

The information access sequence using proposed agencies is as shown in Fig. 5. The numbers in figure denote interaction sequence. It is assumed that information required is available in the vehicle of neighboring cluster. The method to access the information is as given in the following sequence:

1. The vehicle needing information contacts the BSMA through VMA. (BSMA searches information in its CKB and also contacts RTSMA. In this case, BSMA as well as RTSMA does not have the information, hence it informs the VMA.)

2. VMA creates the SDA to its neighboring vehicles.

3. SDA migrates to neighboring vehicle and communicates to VMA through TKB. 


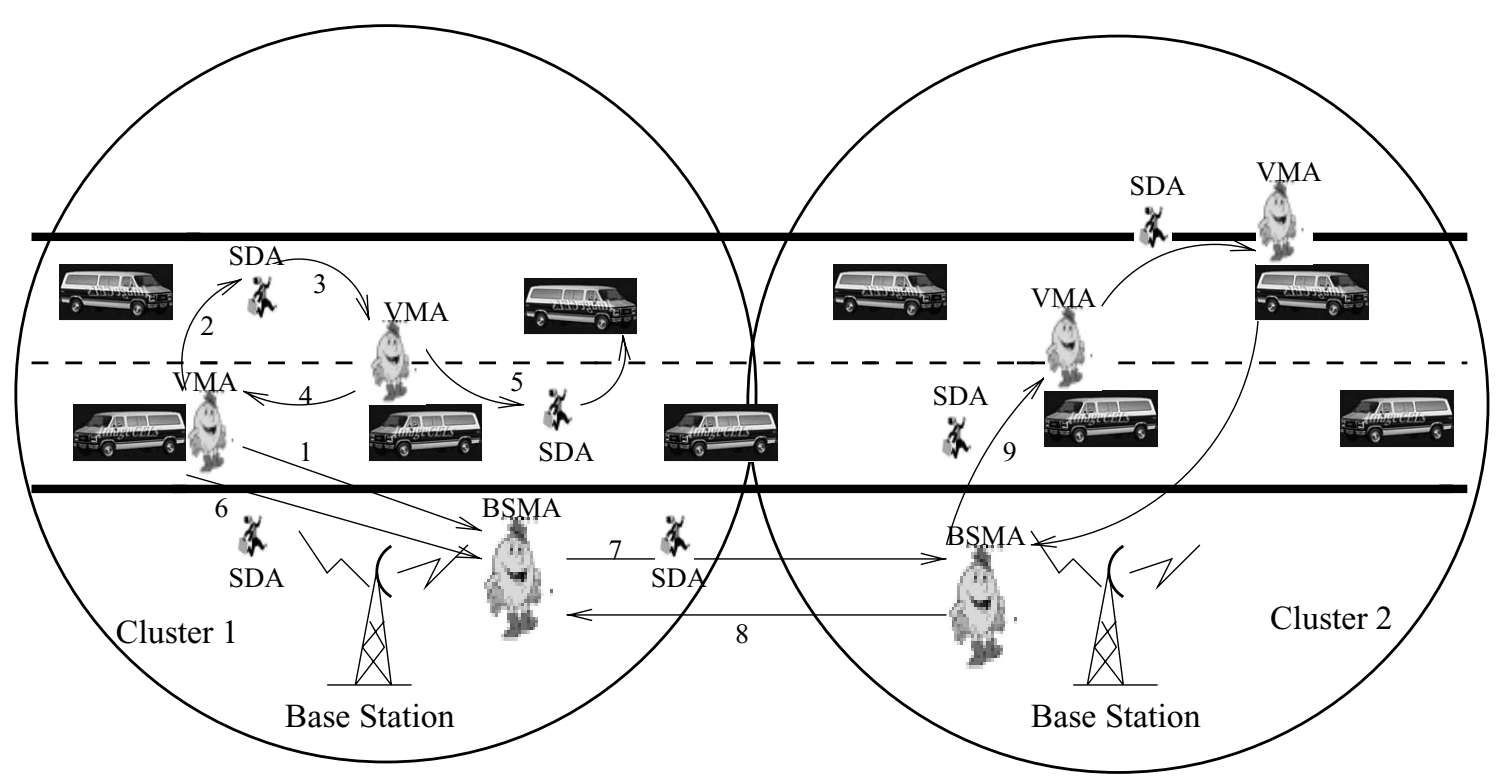

Fig. 5. Information search and access in VANETs.

4. If the required information is available in TKB of the neighboring vehicles SDA sends the information to the VMA.

5. If the information is not available with the neighboring vehicle, SDA clones from its place and moves to second degree neighbors and so on within the cluster. (If it identifies the required information with a particular vehicle then the information will be sent to VMA. SDA and its clone destroy themselves.)

6. If the information is not available within the cluster, VMA again generates SDA which migrates to its base station.

7. SDA clones itself to neighboring clusters by communicating with its cluster BSMA under certain norms.

8. In case SDA gets information at neighboring BSMA, it returns to its created VMA.

9. If SDA fails in getting the information from neighboring BSMAs, it searches within the neighboring cluster vehicles as given in steps 3 to 5 .

\subsubsection{Critical information dissemination}

Search for information, in the above application, is concerned with information pull. In this application, we are concerned with information push, whereby vehicles spread messages about safety related events such as accidents, road conditions (roadworks), inter-vehicle distance, weather conditions ahead, etc., through AA. Critical information related events may be of two kinds. Firstly, there are events (such as fuel status, vehicle speed, neighbor vehicle distance, etc.) that can be detected by an AA for a particular vehicle. These events will assist the driver in safer driving and it does not need to be spread to other vehicles. Secondly, there are events such as traffic jams, accidents, road conditions, etc., which have to be disseminated to other vehicles in an aggregated way. Aggregation requires aggregating the events sensed by a single vehicle as well as aggregating the events of all the vehicles.

The critical information dissemination using proposed model is shown in Fig. 6. The numbers in figure denote interaction sequence. The method to disseminate the critical information is as given in the following sequence: 


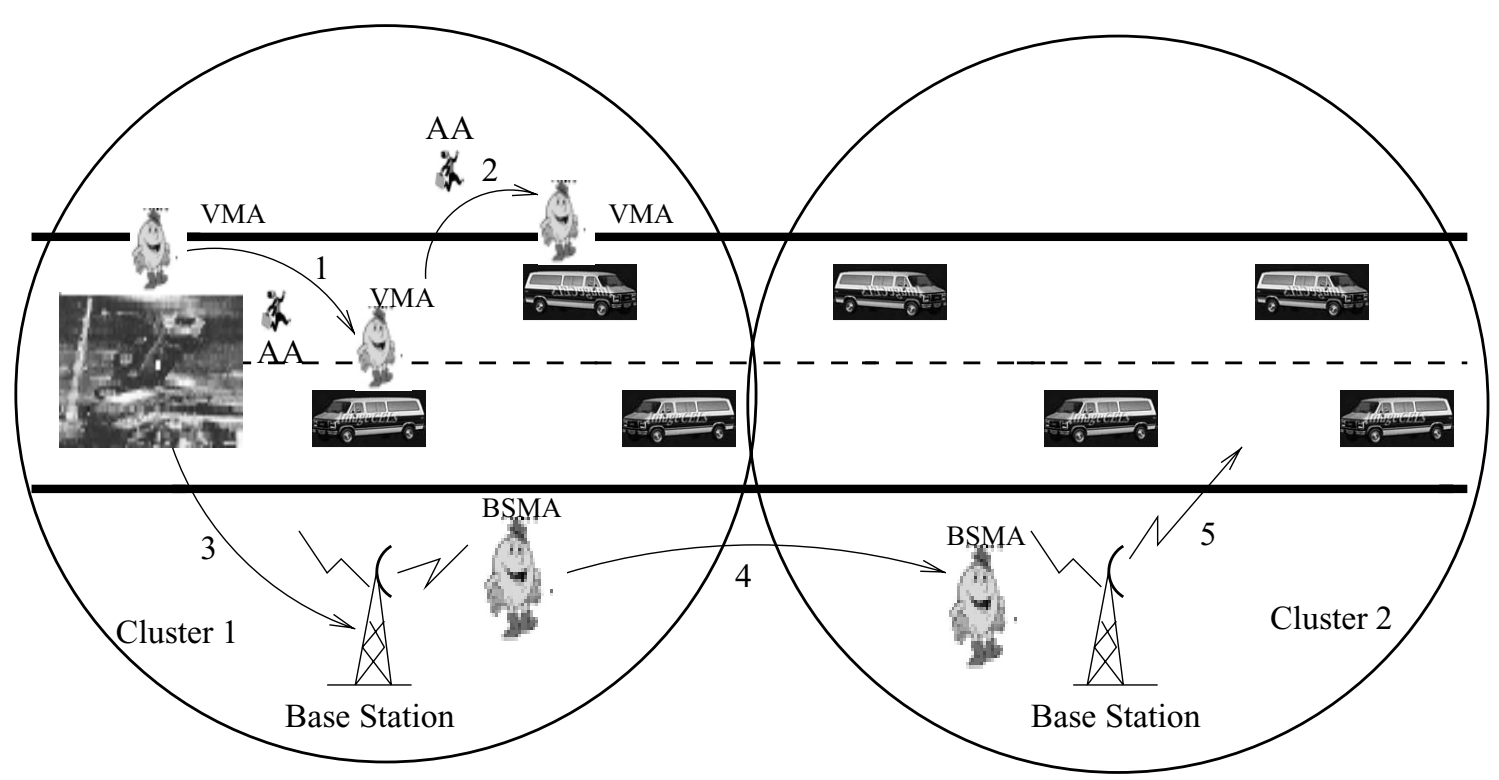

Fig. 6. Critical information dissemination model in VANETs.

1. Whenever critical events occur, VMA in a vehicle creates AA to its neighbors. ( AA communicates with neighboring VMA and informs about the critical event as well as collects any critical information available in the visited vehicle.)

2. The neighboring VMA which received the message of critical event, creates clones of AA and spreads the message to its neighboring vehicle and so on.

3. VMA communicates critical information to its BSMA. (BSMA updates CKB based on certain permitted actions depending on the norms.

4. BSMA communicates about the received critical information to the neighboring BSMAs as well as to RTSMA.

5. Neighboring BSMAs broadcast critical information in its cluster.

\subsubsection{Location-dependent services}

Location-dependent services can be built over the information push-pull model of the two previous scenarios. Certain information such as the location of the nearest facilities like fuel stations, parking zones, entertainment places and restaurants, markets, etc., can be accessed through regional transport station. Figure 7 depicts the information advertisement by using the proposed model. The numbers in figure denote interaction sequence. The method to access local information about roadside (or nearby accessible) services is given in the following sequence:

1. RTS manager sends the information to all BSMAs.

2. BSMA updates its CKB and informs SA to advertise the message through ADAs.

3. SA creates several ADAs which move to the nearest vehicles and pass on the information to users as well as interact to get some or nil response for the advertisement.

4. ADAs clones themselves and visits all the vehicles that are not visited by any other ADA and repeats the operation as given in step 3.

5. Parent ADA accumulates all the responses and sends the information to SA which in turn passes on the information to RTS manager. 


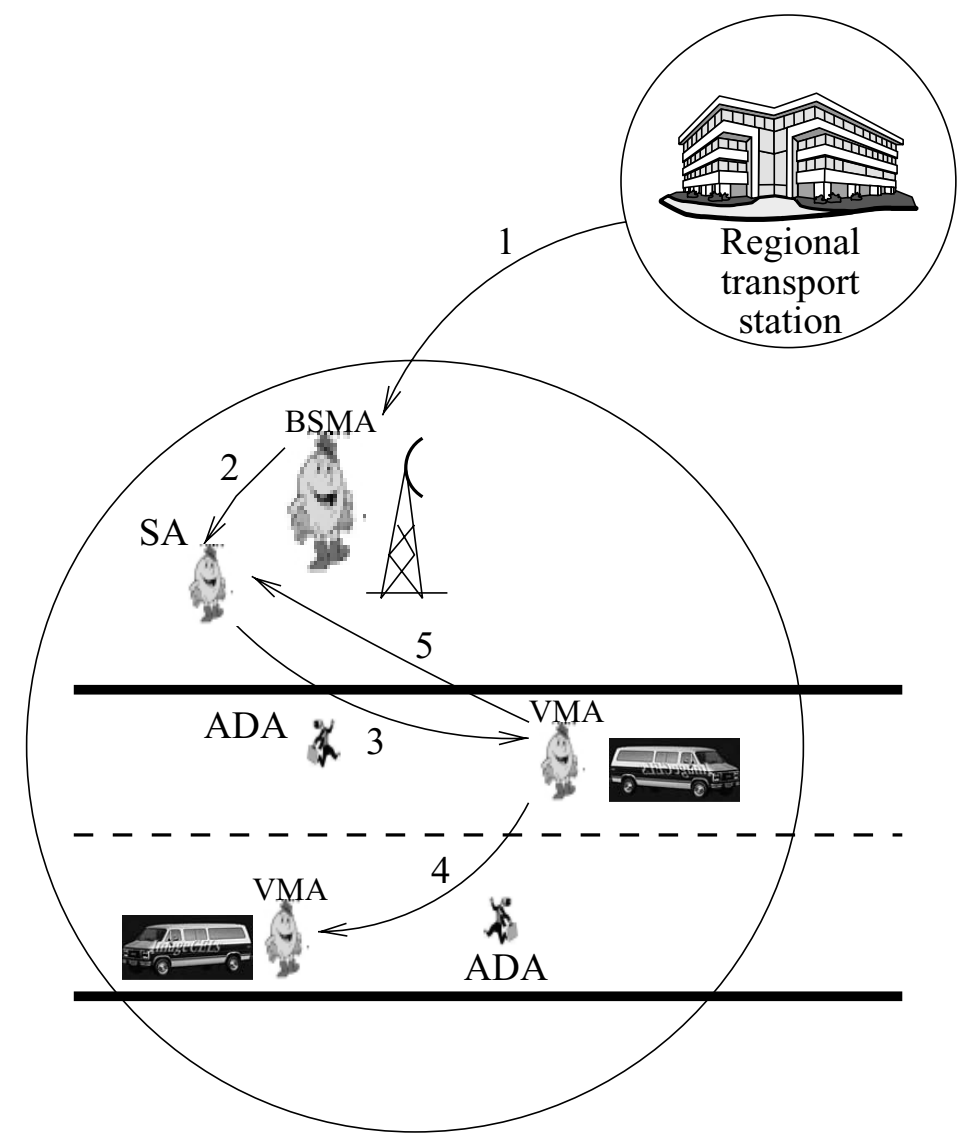

Fig. 7. Advertising information from transport agency.

\section{Simulation}

We have simulated the proposed model by using several network topologies on a Pentium- 4 machine by using $\mathrm{C}++$ programming language to test the performance and effectiveness of the approach with respect to the Bangalore city map. The Bangalore city map which is considered for the simulation is as shown in the Fig. 8. In this section, we discuss the network traffic model, simulation procedure and the results.

\subsection{Network traffic model}

We consider vehicles moving in a fixed region of length $A \mathrm{Km}$. and breadth $B \mathrm{Km}$. $N$ number of base stations located at distant places in Bangalore city are considered. We also consider some of base stations $(U \%)$ as unreliable ones. The communication coverage range for each base station is $C_{r a b}$. The traffic volume for Bangalore city is classified as heavy traffic, medium traffic and low traffic. The timings for heavy, medium and low traffic are $T_{h}, T_{m}$ and $T_{l}$ respectively.

Total number of vehicles generated are $X$ vehicles/second for heavy traffic, $Y$ vehicles/second for medium traffic, and $Z$ vehicles/second for low traffic. These statistics are approximated from 24-hour traffic volume of Bangalore city real traffic data. The border effect of the bounded simulation region on the vehicle mobility is accounted for by making the vehicle reappear in the region. 


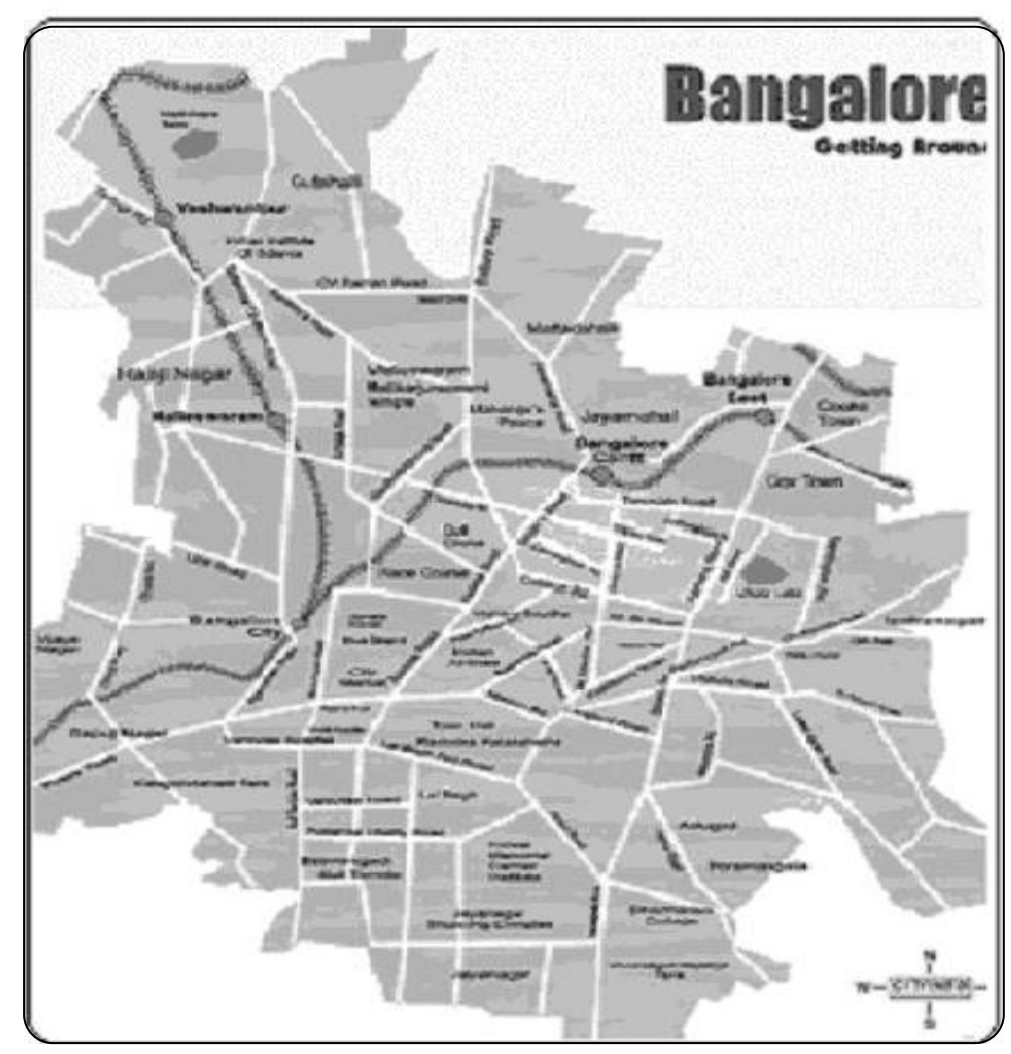

Fig. 8. Bangalore road map.

In order to simulate the mobility of vehicles in this vehicular network, we considered the vehicles to move in a road of the type $R_{\text {type }}$, with the number of lanes $L$, lane of length $P \mathrm{Km}$ and with vehicles moving in only one direction. The lane separation in the road is $Q$ meters. While assigning the mobility for the vehicles, it is assumed that the speed and distance of a succeeding vehicle, by making it to keep a safety distance of $R$ meters from the preceding vehicle for a certain tolerance time, and then change lane if possible. Changing lane allows the vehicle to move to an adjacent lane if there is space (safety distance) in that lane.

Communication medium access protocol considered for the simulation is the Distributed Coordination Function (DCF) of IEEE 802.11 which is responsible for the medium access based on CSMA with Collision Avoidance (CSMA/CA). The protocol uses following parameters: Distributed Inter Frame Space (DIFS), Short Inter Frame Space (SIFS) and a contention window $(C W)$.

At every intersection, we assume that each vehicle can choose to make either a left or right (if not a one-way road) or to no turn. The communication coverage area for each vehicle is considered as a $V_{\text {com }}$ meters. At the beginning of the simulation, the vehicles are uniformly distributed in the lanes. This setting holds under the assumption that there is a free flow movement of vehicles, i.e. we do not account for congestion that may arise in the roads. It is assumed that all the vehicles are equipped with a communication device and knows the start position, start time of the vehicle, the route that it selects, and the speed at which it travels. The mobility factor for each node is in between the range of I to $J \mathrm{Kmph}$ (Kilometers per hour). Services in each node are in the range of $S_{\min }$ to $S_{\max }$. 


\subsection{Simulation procedure}

To illustrate some results of the proposed VANET simulation, we have taken $A=60 \mathrm{Km}$ and $B=60 \mathrm{Km}, N=97, U=00 \%$ to $25 \%, C r a b=2 \mathrm{Km}, T_{h}=9.00$ to 11.00 am and 5.00 to $9.00 \mathrm{pm}, T_{m}=11.00$ am to $5.00 \mathrm{pm}, T_{l}=9.00 \mathrm{pm}$ to $9.00 \mathrm{am}, X=1000$ vehicles/second/lane, $Y=$ 500 vehicles/second/lane, and $Z=200$ vehicles/second/lane, $R_{\text {type }}=$ free way, $L=2, P=5 \mathrm{Km}, Q=$ 5 meters, $R=10$ meters, $C W=15$ slots, $S I F S=32 \mu \mathrm{sec}, D I F S=58 \mu \mathrm{sec}, V_{\text {com }}=100$ meters, $I=0 \mathrm{Kmph}$ and $J=40 \mathrm{Kmph}, S_{\min }=1$ and $S_{\max }=50$.

\section{Begin}

1. Generate the VANET for the given number of vehicles

2. Apply the proposed model

3. Compute the performance of the system

\section{End}

The performance parameters measured are:

- Network configuration time: It is defined as the total amount of time taken by the network to configure under all the base stations for a given number of vehicles.

- Information saturation time: It is the total amount of time taken to disseminate the information about the newly generated event in the network.

- Latency: It is defined as the time required for discovering and acknowledging the requested ser$\operatorname{vice}(\mathrm{s})$.

- Bandwidth Utilization: It is defined as the ratio of the sum of bandwidth utilized by all the clusters (base station coverage area) to the sum of the maximum bandwidth of all the clusters.

- BSMA response time: It is defined as the time taken by the BSMA to service requests of vehicles within its base station range.

\subsection{Results}

Network configuration time increases with increase in the density of vehicles as shown in Fig. 9. Here we have analyzed this result for two cases; (i) 100\% reliable base stations and (ii) $75 \%$ reliable base stations. In the first case, network configuration time is little high and the network overheads increase as compared to second case. In both the cases, network configuration time varies linearly with respect to the variation of the number of vehicles.

From Fig. 10, we observe that information saturation time for $100 \%$ reliable base stations linearly increases. Information saturation time for heavy traffic is very high as compared to low traffic and medium traffic scenarios. During the heavy traffic, high vehicle density leads to high saturation time in order to reach the information to all the vehicles.

Figure 11 shows the information saturation time for $75 \%$ reliable base stations. In this case, for low number of events (up to 6 events) the information saturation time does not show much variation whereas for high number of events generated in the network the information saturation time rapidly varies. We observe that the information saturation time for heavy traffic is very high as compared to low traffic and medium traffic scenarios. It is because, in the absence of base stations, the neighboring cluster vehicles will take the responsibility of propagating the information of new generated event. 


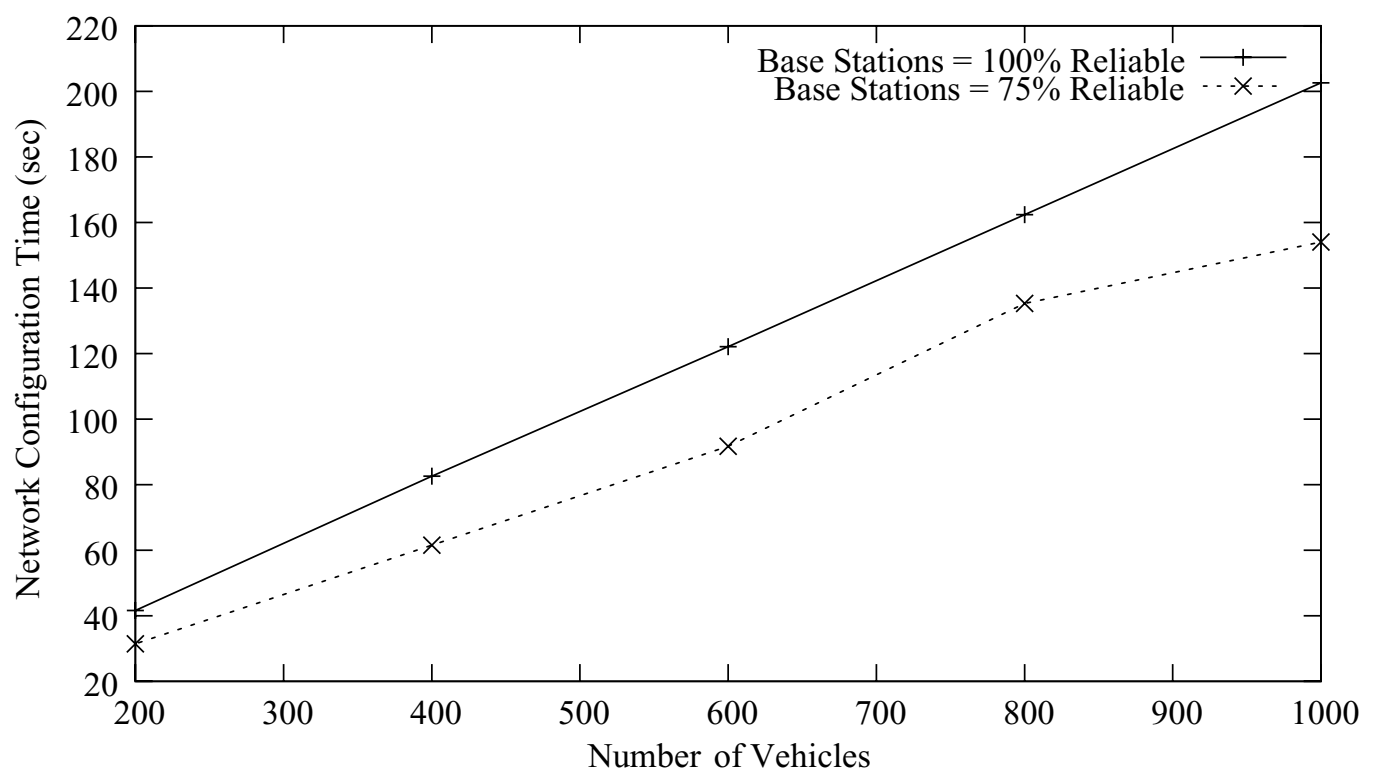

Fig. 9. Network configuration time vs. number of vehicles.

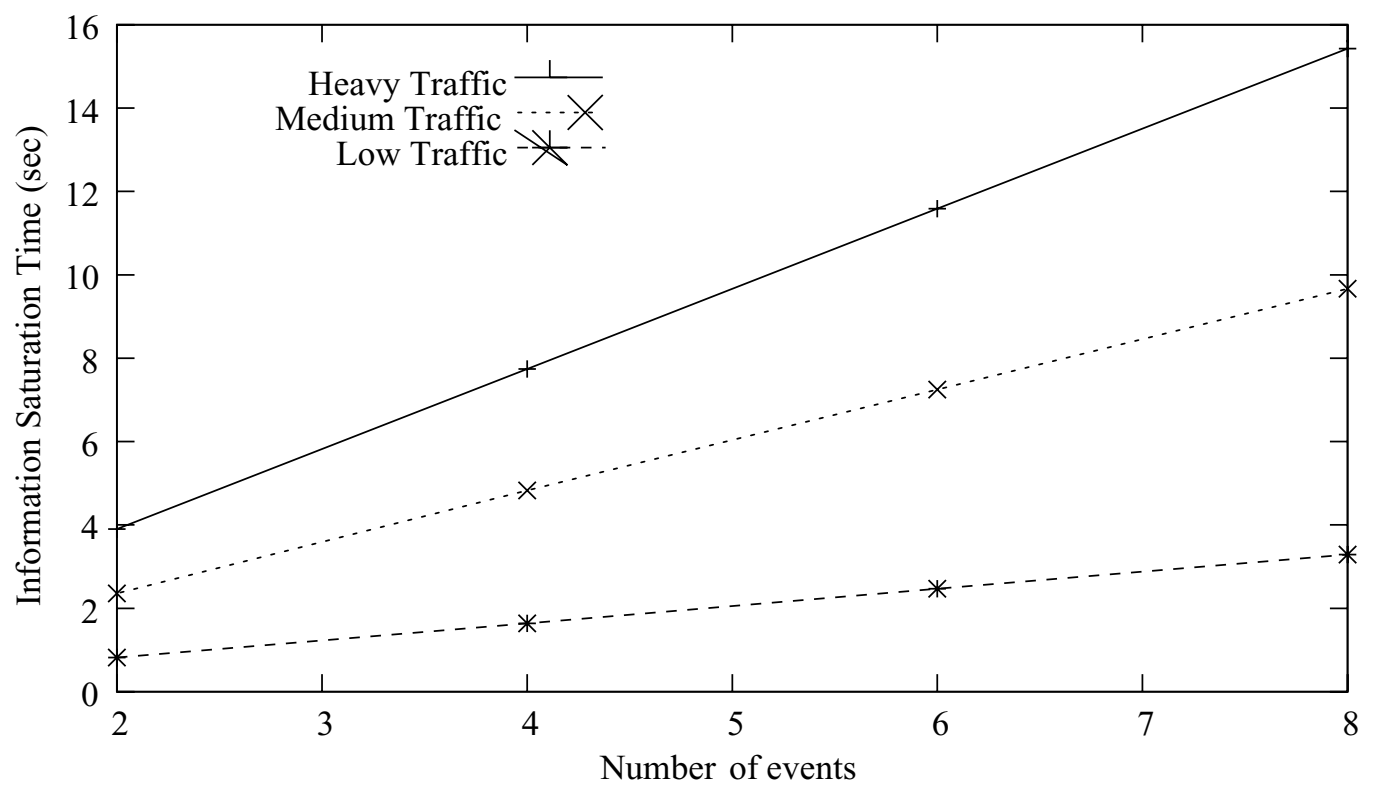

Fig. 10. Information saturation time vs. event generated [100\% reliable base stations].

Figure 12 shows the latency for a given number of services. As the number of vehicles increase the latency is increased for a given number of services. The latency will not show much variations for low traffic and medium traffic scenarios. But, for high traffic scenario the latency is high.

We observe from Fig. 13, that the bandwidth utilization percentage has a linear increase with increase in the number of services. The curves in the graph show the variations for the number of services with 


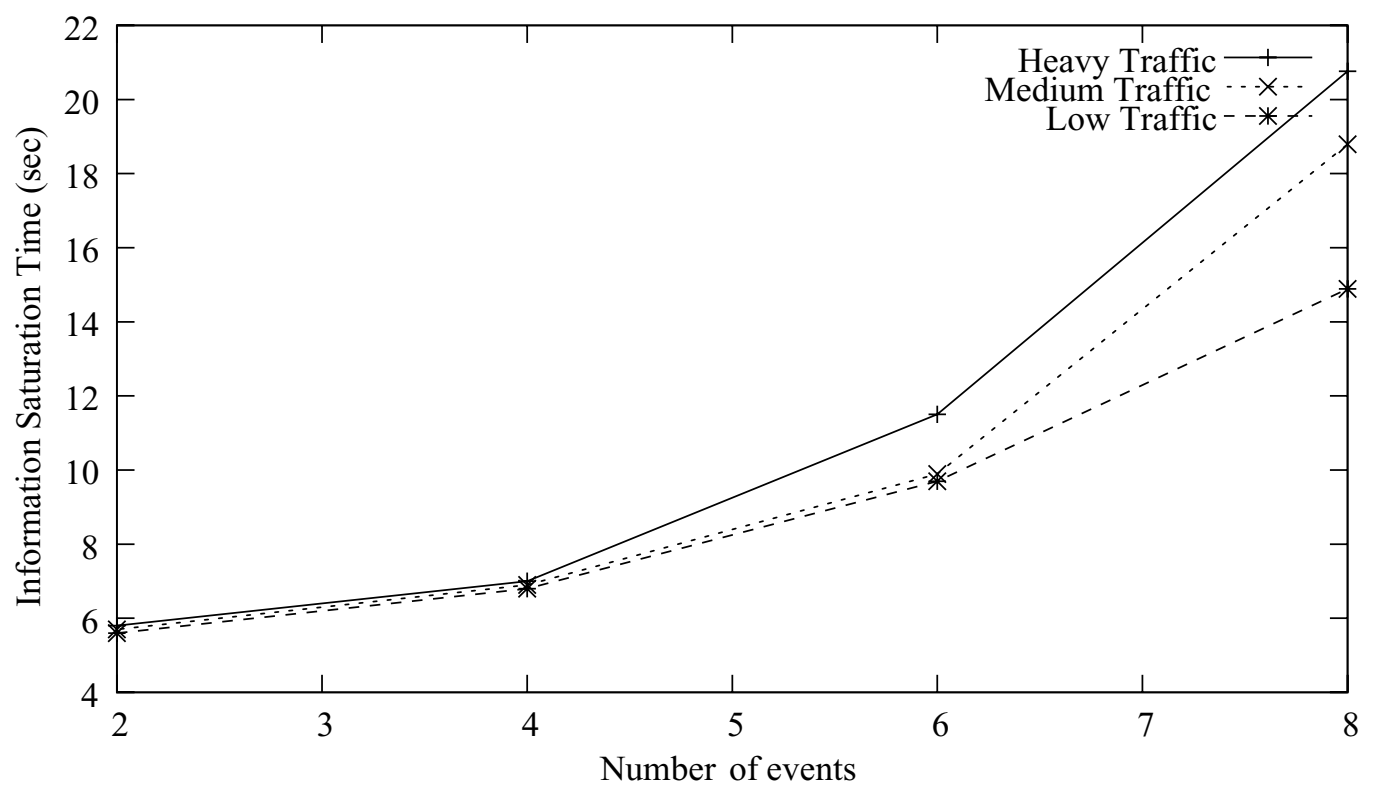

Fig. 11. Information saturation time vs. event generated [75\% reliable base stations].

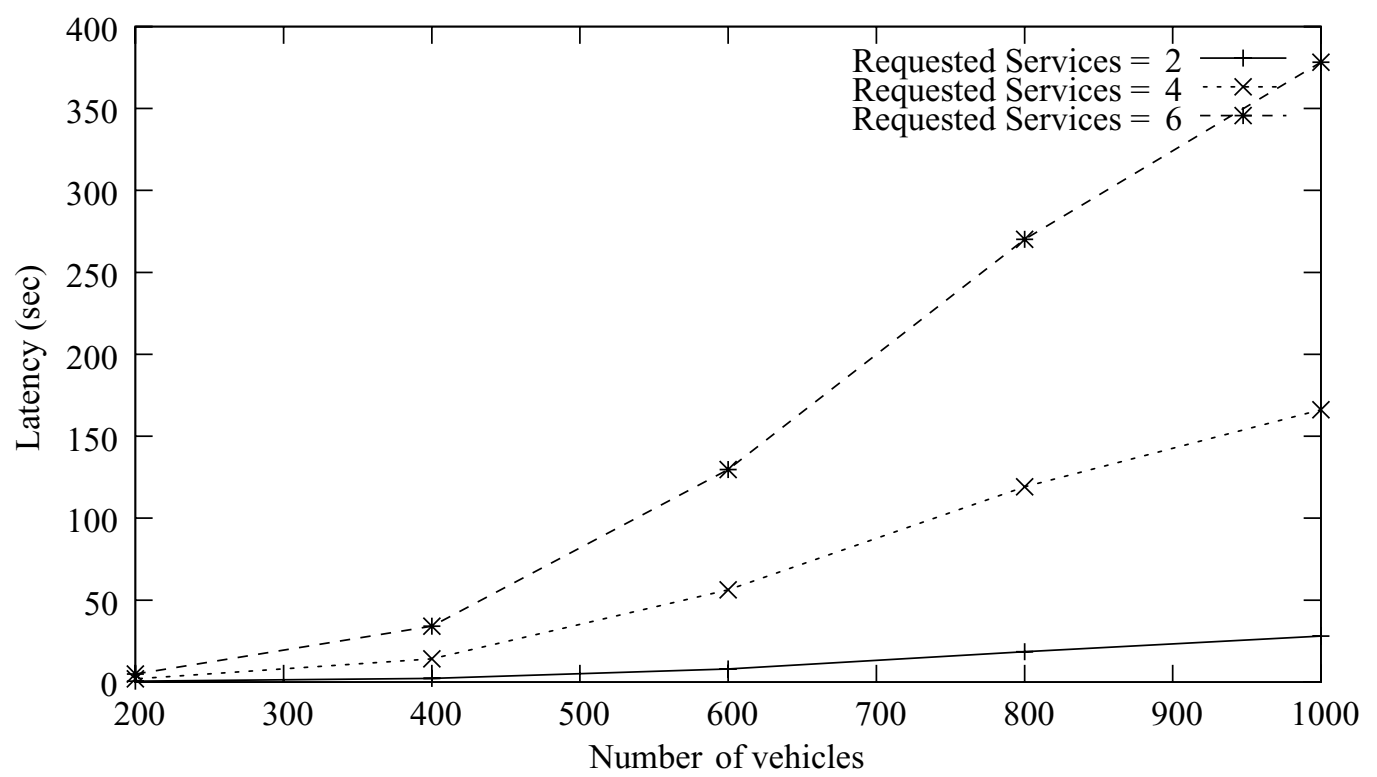

Fig. 12. Latency vs. density of vehicles.

fixed requirement of bandwidth. This performance parameter curve will be very useful in order to get the effective utilization of the available network bandwidth.

As the number of vehicles increase, the response curve for the given number of service linearly increases (see Fig. 14). From this result, we may conclude that the base station response time increases as the number of services increase. 


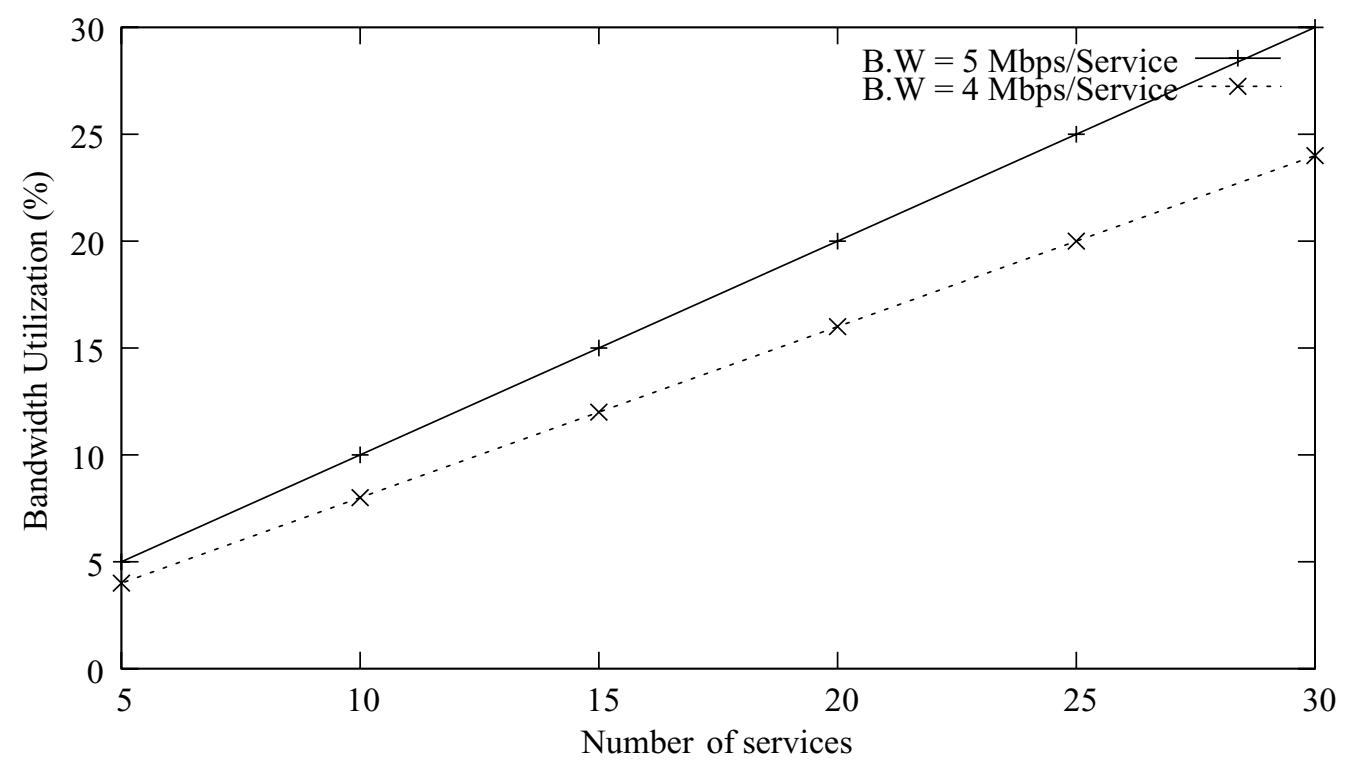

Fig. 13. Bandwidth utilization vs. number of services.

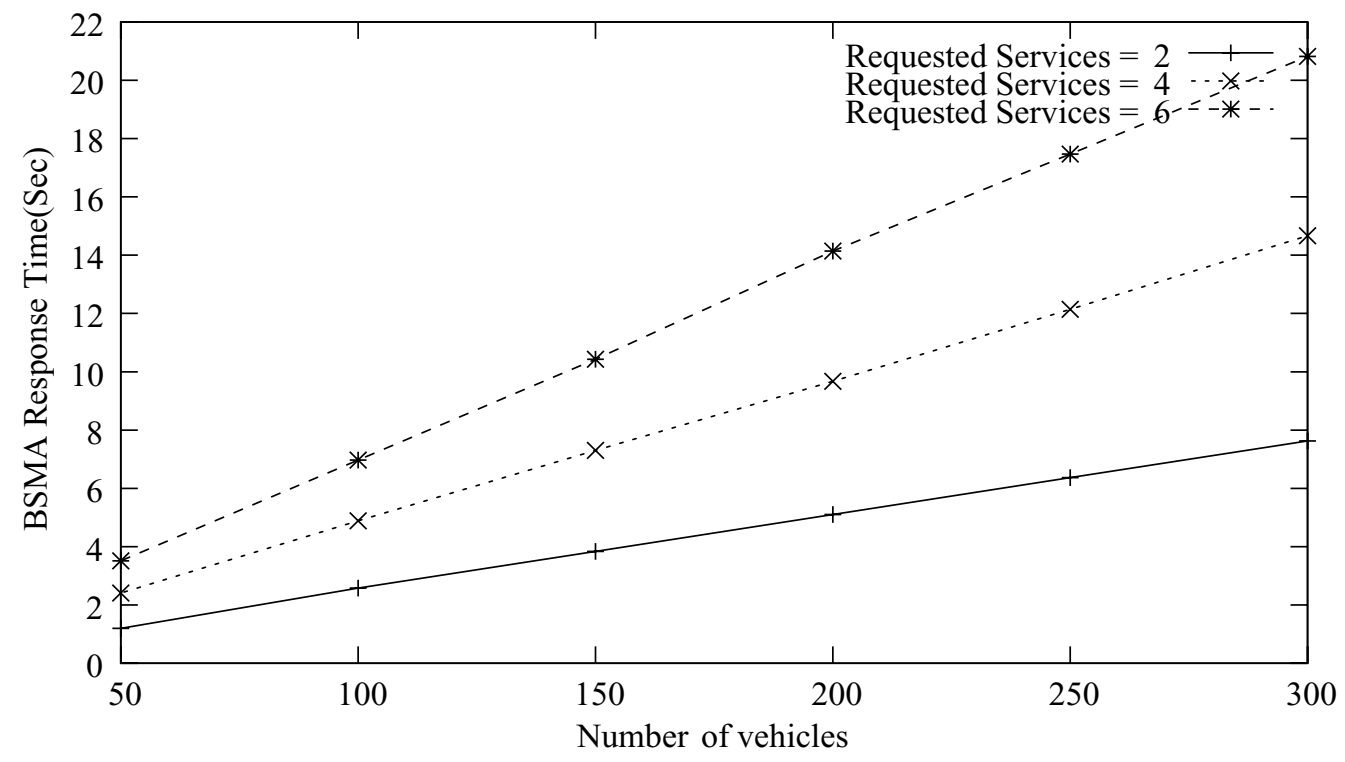

Fig. 14. BSMA response time vs. density of vehicles.

\section{Benefits of using agent technology}

The following are some of the benefits of using agents in the proposed vehicular information ad hoc networks.

- Flexibility: Agents are flexible to accommodate varieties of services to facilitate information dissemination in VANETs. For example, SDA may be encoded to discover multiple services rather 
than single service based on user degree of satisfaction.

- Adaptability: As we observe in the applications mentioned above, we can see that agents such as AA, SDA and ADA adapt to varied network conditions such as vehicle mobility, occurrence of critical events, changes in road and weather conditions, etc.

- Maintainability: The agent-based approach we have advocated is predicated entirely on the idea of open systems, that is, the interaction of heterogeneous and unpredictable components. However, the use of norm aware agents considers situations where design-time specifications may need to be modified at run-time; or where the system specifications may only be partially given at design-time, and the components themselves complete the specifications at run-time.

- Survivability: Wireless networks are specifically designed to operate in the expectation of contention and error. Similarly, in VANETs, it may be that a node fails to comply with the system specifications, either by design, by accident or from necessity. Dealing with such non-compliant behavior, can also be addressed by the norm-governed approach, where appropriate behavior can be stipulated using concepts stemming from the study of legal and social systems: e.g. permissions, obligations and other normative relations such as power, right and entitlement.

\section{Conclusions}

The proposed agent based information services in VANETs identified some of the important issues for information dissemination and proposed a set of novel and effective architecture to resolve them by using agent technology. We developed an agent based model for information services in vehicular ad-hoc networks (VANETs) and analyzed the following performance parameters: network configuration time, information saturation time, latency, and bandwidth utilization and base station response time. An agent based scheme for VANETs exhibited the following benefits: flexible and adaptable services, software reusability and maintainability, and efficient resource utilization.

A further refinement of the network architecture, facilitated by the use of agent technology can be done as follows. Instead of fixed base stations situated at strategic points along the highway defining clusters and vehicles belonging to a cluster according to proximity; we can remove the base stations altogether (with a few exceptions), and propose the logical clusters which physically move the length of the highway, and moving vehicles join or leave clusters according to their ground speed and proximity to identified cluster-heads or gateway nodes. The additional research questions that need to be addressed are: Permanent transience (or transient permanence), Role-based and policy based network management, and Anticipation.

Evaluation of the agent based protocol overheads of the proposed approach which includes the memory and communication overhead, etc. along with the computational overheads and a comparison study of proposed approach with other methods to show the benefits of agent technology in VANETs are considered as the future extension of the work.

\section{Acknowledgments}

We are very much thankful to the reviewers for useful comments that helped us in improving the quality of paper. 


\section{References}

[1] A. Ebner, H. Rohling, M. Lott and R. Halfmann, Decentralized slot synchronization in highly dynamic ad hoc networks, in: 5th International Symposium on Wireless Personal Multimedia Communications (WPMC) 2002, G.L. Stuber, ed., Honolulu, 2002, pp. 494-498.

[2] A. Jones and M. Sergot, On the characterization of law and computer systems: The normative system perspective, in: Deontic Logic in Computer Science: normative system specification, J. Meyer and R. Wieringa, eds, John Wiley UK, 1993, pp. 275-307.

[3] Abdalla M. Hanashi, Irfan Awan, Mike Woodward, Performance evaluation with different mobility models for dynamic probabilistic flooding in MANETs, Mobile Information Systems 5(1), 65-80.

[4] Ardalan Vahidi, and Azim Eskandarian, Research advances in intelligent collision avoidance and adaptive cruise control, IEEE Transactions on Intelligent Transport Systems 4 (2003), 143-153.

[5] B. Reshmi, S.S. Manvi, Bhagyavathi, An agent based intrusion detection model for MANETs, Mobile Information Systems 2 (2006), 169-191.

[6] C. Castelfranchi and E. Lorini, Cognitive anatomy and functions of expectations, in: IJCAIO3 Workshop on Cognitive Modeling of Agents and Multi-Agent Interactions, A.G. Cohn, ed., Morgan Kaufmann Publishers, Acapulco, 2003, pp. 307-311.

[7] E. Fonseca and A. Festag, A survey of existing approaches for secure ad hoc routing and their applicability to VANETS, NEC Technical Report NLE-PR-2006-19, NEC Network Laboratories, 2006.

[8] F. Sailhan and V. Issarny, Scalable service discovery for MANET, in: 3rd IEEE International Conference on Pervasive Computing and Communications, 2005, pp. 235-244.

[9] G. Cabri, L. Leonardi, M. Mamei and F. Zambonelli, Mobile agent organizations, in: Fifth IEEE International Conference on Mobile Agents (MA), Atlanta, 2001.

[10] H. Fubler, M. Moreno, M. Transier, A. Festag and H. Hartenstein, Thoughts on a protocol architecture for vehicular ad-hoc networks, in: 2nd International Workshop in Intelligent Transportation (WIT 2005), Hamburg, 2005, pp. 41-45.

[11] I. Athanasiadis and P. Mitkas, An agent-based intelligent environmental monitoring system, Management of Environmental Quality 15 (2004), 238-249.

[12] J. Bradshaw, Introduction to software agents, AAAI press/The MIT Press, 1997.

[13] J. Jetcheva, Yih-Chun Hu, S. PalChaudhuri et al., Design and evaluation of a metropolitan area multi tier wireless ad hoc network architecture, in: 5th IEEE Workshop on Mobile Computing Systems and Applications (WMCA), R. Kravets, ed., Wiley, John and Sons, Monterey, 2003, pp. 32-43.

[14] J. Pitt, P. Venkataram and A. Mamdani, QoS management in MANETs using norm-governed agent societies, in: 6th International Workshop Engineering societies in the agents world, D. Oguz et al., eds, Springer Verlag, Kusadasi, 2005, pp. 221-240.

[15] J. Chen, S.H.G. Chan and J. He, Soung-Chang Liew, Mixed Mode WLAN: The Integration of Ad Hoc Mode with Wireless LAN Infrastructure, in: IEEE International Conference on Communications (ICC), Korea, 2005.

[16] J.S. Kwak and J.H. Lee, Infrared transmission for inter vehicle ranging and Vehicle-to-Roadside communication systems using spread-spectrum technique, IEEE transactions on intelligent transport systems 5 (2004), 12-19.

[17] J.J. Blum, A. Eskandarian and L.J. Hoffman, Challenges of inter vehicle ad hoc networks, IEEE transactions on intelligent transport systems $\mathbf{5}$ (2004), 347-351.

[18] J. Luo and J.P. Hubaux, A survey of inter-vehicle communication, in: Embedded Security in Cars-Securing Current and Future Automotive IT applications, K. Lemke et al., eds, Springer-Verlag, Leipzig, 2005, pp. 164-179.

[19] K. Zimmermann, L. Egger and M. Brunner, Self-Management of wireless base stations, in: IEEE Workshop on Management Issues and Challenges in Mobile Computing, Nice, 2005, pp. 119-123.

[20] M. Al-kahtani and H. Mouftah, Congestion control and clustering stability in wireless ad hoc networks: Enhancements for clustering stability in mobile ad hoc networks, in: 1st ACM Workshop on Quality of service and security in wireless and mobile networks, 2005, pp. 112-121.

[21] M. Bechler, H.J. Hof, D. Kraft, F. Pahlke and L. Wolf, A cluster based security architecture for ad hoc networks, in: IEEE INFOCOM, Hong Kong, 2004, pp. 172-194.

[22] M. Jakobsson, Xiao Feng Wang and S. Wetzel, Stealth attacks in vehicular technologies, in: First International Workshop on Vehicular Ad hoc Networks, Philadelphia, 2004, pp. 123-129.

[23] M.L. Griss and G. Pour, Development with agent components, IEEE Computer magazine 34 (2001), 37-43.

[24] M. Nekovee, Sensor networks on the road: the promises and challenges of vehicular ad hoc networks and grids, in: Workshop on Ubiquitous Computing and e-Research, Edinburgh, 2005.

[25] M. Rydstrom, A. Toyserkani, E. Strom and A. Svensson, Towards a wireless network for traffic safety applications, in: Radio Science and Communication, Linkoping, 2005, pp. 375-380.

[26] N.R. Jennings, An agent-based approach for building complex software systems, Communications of ACM 44 (2001), $35-41$. 
[27] N.R. Jennings, Developing agent-based systems, IEE Software Engineering 44 (1997), 1-2.

[28] P. Sethi and G. Barua, Dynamic cluster management in ad hoc networks, in: HPC 2002, Bangalore, 2002.

[29] P. Golle, D. Greene and J. Staddon, Detecting and correcting malicious data in VANETs, in: First ACM International Workshop on Vehicular Ad-hoc Networks, ACM Publishers, Philadelphia, 2004, pp. 29-37.

[30] R.B. Muhammad, Range assignment problem on the Steiner tree based topology in ad hoc wireless networks, Mobile Information Systems 5 (1), 53-64.

[31] S. Eichler, F. Dotzer, C. Schwingenschlogl, F. Caro and J. Eberspacher, Secure routing in a vehicular ad hoc network, in: IEEE 60th Vehicular Technology Conference, Los Angeles, 2004, pp. 3339-3343.

[32] S. Funfrocken and F. Mattern, Mobile agents as an architectural concept for Internet based distributed Applications - The WASP project approach, http://citeseer.nj.nec.com/14154.html

[33] S. Franklin and A. Graser, Is it an agent or just a program: A taxonomy for autonomous agents, in: International Workshop on Agent Theories, Architectures and Languages, http://citeseer.nj.nec.com/32780.html.

[34] S. Goel, T. Imielinski and K. Ozbay, Ascertaining viability of Wi-Fi based Vehicle-to-Vehicle network for traffic information Dissemination, in: 7th Annual Intelligent Transportation System Conference, Washington, 2004, pp. 10861091.

[35] S. Jaap, M. Bechler and L. Wolf, Evaluation of routing protocols for vehicular ad hoc networks in city traffic scenarios, in: 11th Open European Summer School EUNICE, Colmenarejo, 2005.

[36] S. Russell and P. Norvig, Artificial intelligence a modern approach, Prentice Hall, 1995.

[37] Stefan K, Gehrig and F.J. Stein, Collision avoidance for vehicle following systems, IEEE transactions on intelligent transport systems $\mathbf{8}$ (2007), 1-12.

[38] S.S. Manvi and P. Venkataram, An agent based adaptive bandwidth allocation scheme for multimedia application, in: Journal of systems and software 75 (2005), 305-318.

[39] S.S. Manvi and P. Venkataram, Applications of agent technology in communications: A review, Computer communications 27 (2004), 1493-1508.

[40] S.S. Manvi and M.S. Kakkasageri, Issues in mobile ad hoc networks for vehicular communication, IETE Technical Review 25 (2008), 59-72.

[41] S.S. Manvi, M.S. Kakkasageri and J. Pitt, Information search and access in vehicular ad-hoc networks (VANETs): An agent based approach, in: International Conference on Communication in Computing (CIC-2007), The 2007 World Congress in Computer Science, Computer Engineering, and Applied Computing, H.R. Arabnia, ed., Las Vegas, 2007, pp. 23-29.

[42] S.S. Manvi, M.S. Kakkasageri, J. Pitt, Alex Rathmell, Multi agent systems as a platform for VANETs, in: International Conference on Autonomous Agents and Multiagent Systems (AAMAS) 06, (Agents in Traffic and Transportation- (ATT)), H. Nakashima, ed., ACM publishers, Hakodate, 2006, pp. 35-42.

[43] S. Schmidt and A. Scott, QoS support within active LARA++ routers, http://citeseer.nj.nec.com/schmid00qos.html.

[44] T. Magedanz, K. Rothermel and S. Krause, Intelligent agents:an emerging technology for next generation telecommunications? in: IEEE INFOCOM, IEEE Computer Society Press, San Francisco, 1996, pp. 24-28.

[45] T.D.C. Little, Ashish Agarwal, An information propagation scheme for VANETs, in: 8th International IEEE Conference on Intelligent Transportation Systems, A. Broggi, ed., Vienna, 2005, pp. 155-160.

[46] U. Lee et al., MOBEYES: Smart mobs for urban monitoring with a vehicular sensor network, IEEE Wireless Communications 13 (2006), 52-57.

Sunilkumar S. Manvi received Ph.D from Indian Institute of Science, Bangalore, India. Presently, he is serving as a Professor and Head of Department of Electronics and Communication Engineering, REVA Institute of Technology and Management, Bangalore. His areas of research include wireless/wired networks, AI applications in network management, E-commerce, Grid Computing and multimedia communications. He has published over 30 papers in referred national/international Journals and 90 papers in referred national/international conferences. He has coauthored books "Communication Protocol Engineering" and "Computer Concepts and C Programming" published by PHI. He is a reviewer of several reputed international/national Journals. He has executed several funded projects in wireless networks from AICTE, New Delhi. He is a Board of Studies and Board of Examiners Member of several universities in India. He is a member of IEEE USA, Fellow of IETE, India, Fellow of IE, India and member of ISTE, India. He has been included in Marquifls Whofls Who in World and International Biographies of Cambridge, London in the year 2006.

Mahabaleshwar S. Kakkasageri completed his M.Tech from Visvesvaraya Technological University Belgaum, India. He is pursuing his $\mathrm{PhD}$ in the area of Vehicular Ad hoc Networks (VANETs). Presently he is serving as a Assistant Professor of Department of Electronics and Communication Engineering, Basaveshwara Engineering College, Bagalkot, India. He has published several papers in referred Journals and conferences. He is a student member of IEEE, USA. He has been included in Marqui's Who's Who in World 2008. 
Jeremy Pitt received Ph.D from Imperial College, London. He is serving as a Reader in Department of Electrical and Electronic Engineering, Imperial College, London. His research interests include application of Multi-Agent Systems, especially in communications, Affective Computing and Computer-Mediated Communications. He has published over more than 150 papers in reputed Journals and Conferences. He has been reviewer of several international journals and conferences. He has completed several projects funded by EPSRC and other research organizations such as Emotional Wardrobe, HUMANE (Human Machine Interaction on Emotion), ALFEBIITE (A Logical Framework for Ethical Behaviour between Infohabitants in the Information Trading Economy of the Universal Information Ecosystem), LiMe (Living Memory), etc. He is a member of IEEE, USA, and member of IEE, UK. 

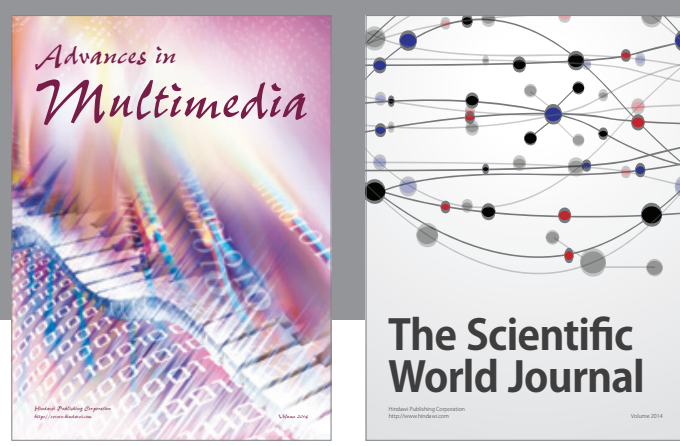

The Scientific World Journal
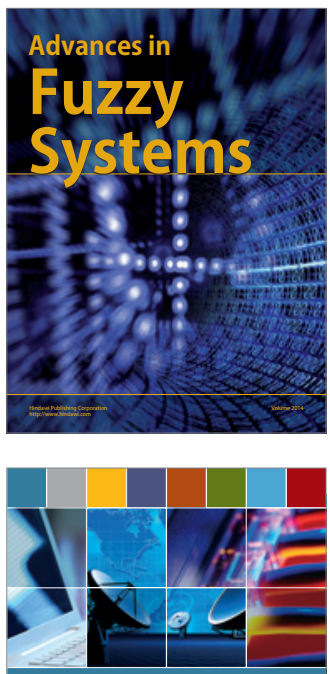

Computer Networks and Communications
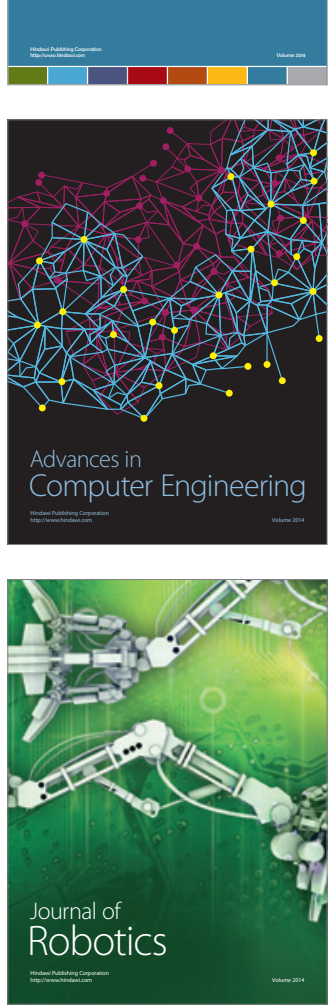
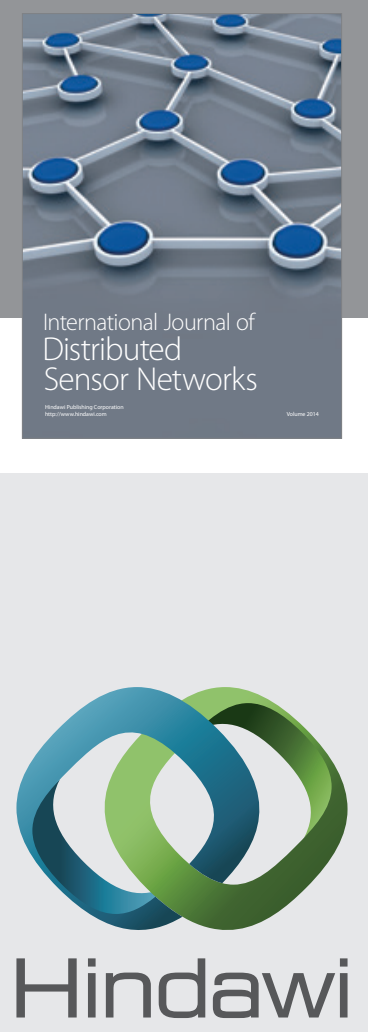

Submit your manuscripts at

http://www.hindawi.com
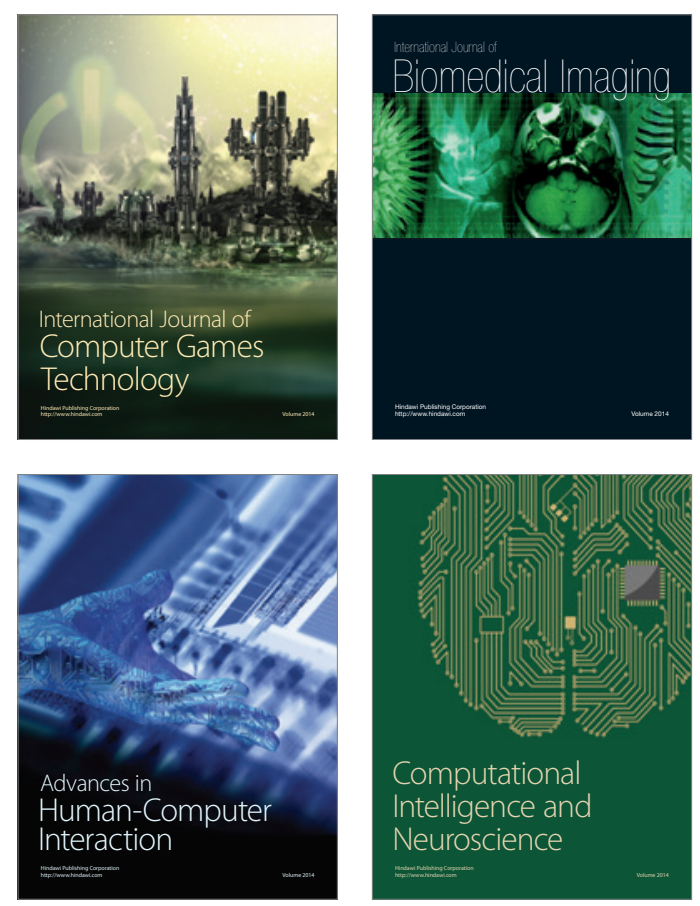
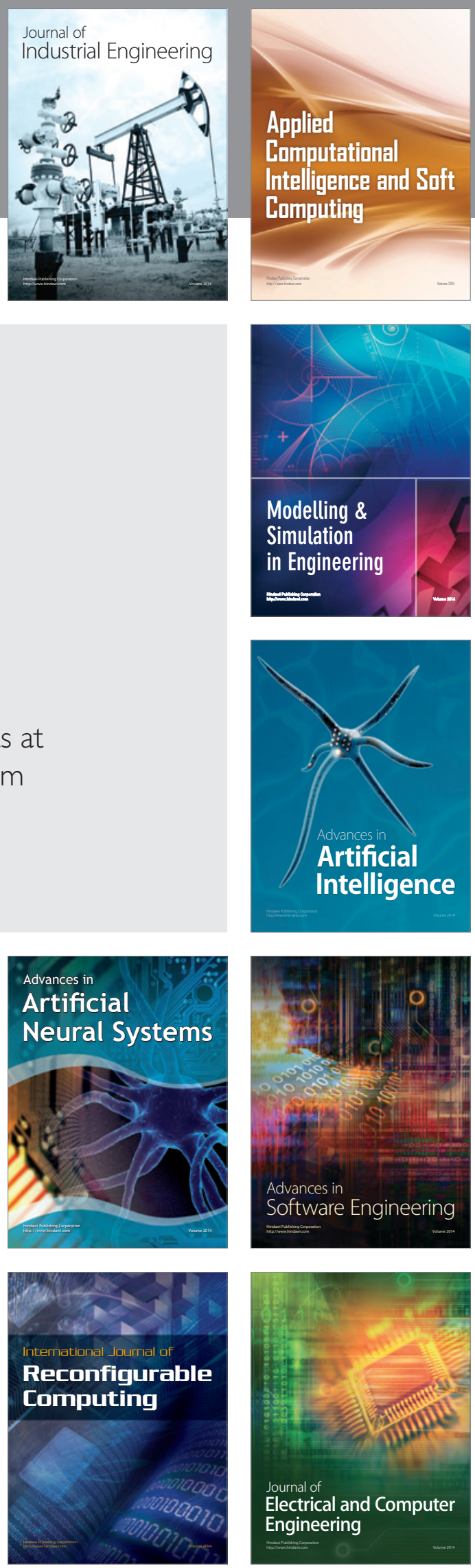